Article

\title{
Some Results on the Graph Theory for Complex Neutrosophic Sets
}

\author{
Shio Gai Quek ${ }^{1, *}$, Said Broumi ${ }^{2}$, Ganeshsree Selvachandran ${ }^{3}$ (1) , Assia Bakali ${ }^{4}$, \\ Mohamed Talea ${ }^{2}$ and Florentin Smarandache 5 (D) \\ 1 A-Level Academy, UCSI College KL Campus, Lot 12734, Jalan Choo Lip Kung, Taman Taynton View, \\ Cheras 56000, Kuala Lumpur, Malaysia \\ 2 Laboratory of Information Processing, Faculty of Science Ben M'Sik, University Hassan II, B.P 7955 \\ Sidi Othman, Casablanca, Morocco; broumisaid78@gmail.com (S.B.); taleamohamed@yahoo.fr (M.T.) \\ 3 Department of Actuarial Science and Applied Statistics, Faculty of Business \& Information Science, \\ UCSI University, Jalan Menara Gading, Cheras 56000, Kuala Lumpur, Malaysia; ganeshsree86@yahoo.com \\ 4 Ecole Royale Navale, Boulevard Sour Jdid, B.P 16303 Casablanca, Morocco; assiabakali@yahoo.fr \\ 5 Department of Mathematics, University of New Mexico, 705 Gurley Avenue, Gallup, NM 87301, USA; \\ fsmarandache@gmail.com or smarand@unm.edu \\ * Correspondence: quekshg@yahoo.com or quekshg@ucsicollege.edu.my; Tel.: +60-17-5008359
}

Received: 25 February 2018; Accepted: 21 May 2018; Published: 31 May 2018

\begin{abstract}
Fuzzy graph theory plays an important role in the study of the symmetry and asymmetry properties of fuzzy graphs. With this in mind, in this paper, we introduce new neutrosophic graphs called complex neutrosophic graphs of type 1 (abbr. CNG1). We then present a matrix representation for it and study some properties of this new concept. The concept of CNG1 is an extension of the generalized fuzzy graphs of type 1 (GFG1) and generalized single-valued neutrosophic graphs of type 1 (GSVNG1). The utility of the CNG1 introduced here are applied to a multi-attribute decision making problem related to Internet server selection.
\end{abstract}

Keywords: complex neutrosophic set; complex neutrosophic graph; fuzzy graph; matrix representation

\section{Introduction}

Smarandache [1] introduced a new theory called neutrosophic theory, which is basically a branch of philosophy that focuses on the origin, nature, and scope of neutralities and their interactions with different ideational spectra. On the basis of neutrosophy, Smarandache defined the concept of a neutrosophic set (NS) which is characterized by a degree of truth membership $T$, a degree of indeterminacy membership $I$, and a degree of falsity membership $F$. The concept of neutrosophic set theory generalizes the concept of classical sets, fuzzy sets by Zadeh [2], intuitionistic fuzzy sets by Atanassov [3], and interval-valued fuzzy sets by Turksen [4]. In fact, this mathematical tool is apt for handling problems related to imprecision, indeterminacy, and inconsistency of data. The indeterminacy component present in NSs is independent of the truth and falsity membership values. To make it more convenient to apply NSs to real-life scientific and engineering problems, Smarandache [1] proposed the single-valued neutrosophic set (SVNS) as a subclass of neutrosophic sets. Later on, Wang et al. [5] presented the set-theoretic operators and studied some of the properties of SVNSs. The NS model and its generalizations have been successfully applied in many diverse areas, and these can be found in [6].

Graphs are among the most powerful and convenient tools to represent information involving the relationship between objects and concepts. In crisp graphs, two vertices are either related or not related to one another so, mathematically, the degree of relationship is either 0 or 1 . In fuzzy graphs on the other hand, the degree of relationship takes on values from the interval $[0,1]$. Subsequently, 
Shannon and Atanassov [7] defined the concept of intuitionistic fuzzy graphs (IFGs) using five types of Cartesian products. The concept fuzzy graphs and their extensions have a common property that each edge must have a membership value of less than, or equal to, the minimum membership of the nodes it connects.

In the event that the description of the object or their relations or both is indeterminate and inconsistent, it cannot be handled by fuzzy, intuitionistic fuzzy, bipolar fuzzy, vague, or interval-valued fuzzy graphs. For this reason, Smarandache [8] proposed the concept of neutrosophic graphs based on the indeterminacy $(I)$ membership values to deal with such situations. Smarandache $[9,10]$ then gave another definition for neutrosophic graph theory using the neutrosophic truth-values $(T, I, F)$ and constructed three structures of neutrosophic graphs: neutrosophic edge graphs, neutrosophic vertex graphs and neutrosophic vertex-edge graphs. Subsequently, Smarandache [11] proposed new versions of these neutrosophic graphs, such as the neutrosophic off graph, neutrosophic bipolar graph, neutrosophic tripolar graph, and neutrosophic multipolar graph. Presently, works on neutrosophic vertex-edge graphs and neutrosophic edge graphs are progressing rapidly. Broumi et al. [12] combined the SVNS model and graph theory to introduce certain types of SVNS graphs (SVNG), such as strong SVNG, constant SVNG, and complete SVNG, and proceeded to investigate some of the properties of these graphs with proofs and examples. Broumi et al. [13] then introduced the concept of neighborhood degree of a vertex and closed neighborhood degree of a vertex in SVNG as a generalization of the neighborhood degree of a vertex and closed neighborhood degree of a vertex found in fuzzy graphs and intuitionistic fuzzy graphs. In addition, Broumi et al. [14] proved a necessary and sufficient condition for a SVNG to be an isolated SVNG.

Recently, Smarandache [15] initiated the idea of the removal of the edge degree restriction for fuzzy graphs, intuitionistic fuzzy graphs and SVNGs. Samanta et al. [16] proposed a new concept called generalized fuzzy graphs (GFG) and defined two types of GFG. Here the authors also studied some of the major properties of GFGs, such as the completeness and regularity of GFGs, and verified the results. In [16], the authors claim that fuzzy graphs and their extensions are limited to the representations of only certain systems, such as social networks. Broumi et al. [17] then discussed the removal of the edge degree restriction of SVNGs and presented a new class of SVNG, called generalized SVNG of type 1, which is an extension of generalized fuzzy graphs of type 1 proposed in [16]. Since the introduction of complex fuzzy sets (CFSs) by Ramot et al. in [18], several new extensions of CFSs have been proposed in literature [19-25]. The latest model related to CFS is the complex neutrosophic set (CNS) model which is a combination of CFSs [18] and complex intuitionistic fuzzy sets [21] proposed by Ali and Smarandache [26]. The CNS model is defined by three complex-valued membership functions which represent the truth, indeterminate, and falsity components. Therefore, a complex-valued truth membership function is a combination of the traditional truth membership function with the addition of the phase term. Similar to fuzzy graphs, complex fuzzy graphs (CFG) introduced by Thirunavukarasu et al. [27] have a common property that each edge must have a membership value of less than or equal to the minimum membership of the nodes it connects.

In this paper, we extend the research works mentioned above, and introduce the novel concept of type 1 complex neutrosophic graphs (CNG1) and a matrix representation of CNG1. To the best of our knowledge, there is no research on CNGs in the literature at present. We also present an investigation pertaining to the symmetric properties of CNG1 in this paper. In the study of fuzzy graphs, a symmetric fuzzy graph refers to a graph structure with one edge (i.e., two arrows on opposite directions) or no edges, whereas an asymmetric fuzzy graph refers to a graph structure with no arcs or only one arc present between any two vertices. Motivated by this, we have dedicated an entire section in this paper (Section 7) to study the symmetric properties of our proposed CNG1.

The remainder of this paper is organized as follows: in Section 2, we review some basic concepts about NSs, SVNSs, CNSs, and generalized SVNGs of type 1; in Section 3, the formal definition of CNG1 is introduced and supported with illustrative examples; in Section 4 a representation matrix of CNG1 is introduced; some advanced theoretical results pertaining to our CNG1 is presented in Section 5, 
followed by an investigation on the shortest CNG1 in Section 6; the symmetric properties of ordinary simple CNG1 is presented in Section 7; and Section 8 outlines the conclusion of this paper and suggests directions for future research. This is followed by the acknowledgments and the list of references.

\section{Preliminaries}

In this section, we present brief overviews of NSs, SVNSs, SVNGs, and generalize fuzzy graphs that are relevant to the present work. We refer the readers to $[1,5,17,18,27]$ for further information related to these concepts.

The key feature that distinguishes the NS from the fuzzy and intuitionistic fuzzy set (IFS) models is the presence of the indeterminacy membership function. In the NS model the indeterminacy membership function is independent from the truth and falsity membership functions, and we are able to tell the exact value of the indeterminacy function. In the fuzzy set model this indeterminacy function is non-existent, whereas in the IFS model, the value of the indeterminacy membership function is dependent on the values of the truth and falsity membership functions. This is evident from the structure of the NS model in which $T+I+F \leq 3$, whereas it is $T+F \leq 1$ and $I=1-T-F$ in the IFS model. This structure is reflective of many real-life situations, such as in sports (wining, losing, draw), voting (for, against, abstain from voting), and decision-making (affirmative, negative, undecided), in which the proportions of one outcome is independent of the others. The NS model is able to model these situations more accurately compared to fuzzy sets and IFSs as it is able to determine the degree of indeterminacy from the truth and falsity membership function more accurately, whereas this distinction cannot be done when modelling information using the fuzzy sets and IFSs. Moreover, the NS model has special structures called neutrosophic oversets and neutrosophic undersets that were introduced by Smarandache in [11], in which the values of the membership functions can exceed 1 or be below 0 , in order to cater to special situations. This makes the NS more flexible compared to fuzzy sets and IFSs, and gives it the ability to cater to a wider range of applications. The flexibility of this model and its ability to clearly distinguish between the truth, falsity, and indeterminacy membership functions served as the main motivation to study a branch of graph theory of NSs in this paper. We refer the readers to $[28,29]$ for more information on the degree of dependence and independence of neutrosophic sets, and [11] for further information on the concepts of neutrosophic oversets and undersets.

Definition 1 [1]. Let $X$ be a space of points and let $x \in X$. A neutrosophic set $A \in X$ is characterized by a truth membership function $T$, an indeterminacy membership function $I$, and a falsity membership function $F$. The values of $T, I, F$ are real standard or nonstandard subsets of $]^{-} 0,1^{+}[\text {, and } T, I, F: X \rightarrow]^{-} 0,1^{+}[. A$ neutrosophic set can therefore be represented as:

$$
A=\left\{\left(x, T_{A}(x), I_{A}(x), F_{A}(x)\right): x \in X\right\}
$$

Since $T, I, F \in[0,1]$, the only restriction on the sum of $T, I, F$ is as given below:

$$
{ }^{-} 0 \leq T_{A}(x)+I_{A}(x)+F_{A}(x) \leq 3^{+}
$$

Although theoretically the NS model is able to handle values from the real standard or non-standard subsets of $]^{-} 0,1^{+}$, it is often unnecessary or computationally impractical to use values from this non-standard range when dealing with real-life applications. Most problems in engineering, and computer science deal with values from the interval $[0,1]$ instead of the interval $]^{-} 0,1^{+}[$, and this led to the introduction of the single-valued neutrosophic set (SVNS) model in [5]. The SVNS model is a special case of the general NS model in which the range of admissible values are from the standard interval of $[0,1]$, thereby making it more practical to be used to deal with most real-life problems. The formal definition of the SVNS model is given in Definition 2. 
Definition 2 [5]. Let $X$ be a space of points (objects) with generic elements in $X$ denoted by $x$. A single-valued neutrosophic set $A$ (SVNS $A$ ) is characterized by a truth-membership function $T_{A}(x)$, an indeterminacy-membership function $I_{A}(x)$, and a falsity-membership function $F_{A}(x)$. For each point $x \in X$, $T_{A}(x), I_{A}(x), F_{A}(x) \in[0,1]$. The SVNS A can therefore be written as:

$$
A=\left\{\left(x, T_{A}(x), I_{A}(x), F_{A}(x)\right): x \in X\right\}
$$

Definition 3 [26]. Denote $i=\sqrt{-1}$. A complex neutrosophic set $A$ defined on a universe of discourse $X$, which is characterized by a truth membership function $T_{A}(x)$, an indeterminacy-membership function $I_{A}(x)$, and a falsity-membership function $F_{A}(x)$ that assigns a complex-valued membership grade to $T_{A}(x), I_{A}(x), F_{A}(x)$ for any $x \in X$. The values of $T_{A}(x), I_{A}(x), F_{A}(x)$ and their sum may be any values within a unit circle in the complex plane and is therefore of the form $T_{A}(x)=p_{A}(x) e^{i \mu_{A}(x)}, I_{A}(x)=q_{A}(x) e^{i v_{A}(x)}$, and $F_{A}(x)=r_{A}(x) e^{i \omega_{A}(x)}$. All the amplitude and phase terms are real-valued and $p_{A}(x), q_{A}(x), r_{A}(x) \in[0,1]$, whereas $\mu_{A}(x), v_{A}(x), \omega_{A}(x) \in(0,2 \pi]$, such that the condition:

$$
0 \leq p_{A}(x)+q_{A}(x)+r_{A}(x) \leq 3
$$

is satisfied. A complex neutrosophic set A can thus be represented in set form as:

$$
A=\left\{\left\langle x, T_{A}(x)=a_{T}, I_{A}(x)=a_{I}, F_{A}(x)=a_{F}\right\rangle: x \in X\right\},
$$

where $T_{A}: X \rightarrow\left\{a_{T}: a_{T} \in C,\left|a_{T}\right| \leq 1\right\}, I_{A}: X \rightarrow\left\{a_{I}: a_{I} \in C,\left|a_{I}\right| \leq 1\right\}, F_{A}: X \rightarrow\left\{a_{F}: a_{F} \in C,\left|a_{F}\right| \leq 1\right\}$, and also:

$$
\left|T_{A}(x)+I_{A}(x)+F_{A}(x)\right| \leq 3 .
$$

Definition 4 [26]. Let $A=\left\{\left(x, T_{A}(x), I_{A}(x), F_{A}(x)\right): x \in X\right\}$ and $B=\left\{\left(x, T_{B}(x), I_{B}(x), F_{B}(x)\right): x \in X\right\}$ be two CNSs in $X$. The union and intersection of $A$ and $B$ are as defined below.

(i) The union of $A$ and $B$, denoted as $A \cup_{N} B$, is defined as:

$$
A \cup_{N} B=\left\{\left(x, T_{A \cup B}(x), I_{A \cup B}(x), F_{A \cup B}(x)\right): x \in X\right\},
$$

where $T_{A \cup B}(x), I_{A \cup B}(x), F_{A \cup B}(x)$ are given by:

$$
\begin{aligned}
T_{A \cup B}(x) & =\max \left(p_{A}(x), p_{B}(x)\right) \cdot e^{i \mu_{A \cup B}(x)}, \\
I_{A \cup B}(x) & =\min \left(q_{A}(x), q_{B}(x)\right) \cdot e^{i v_{A \cup B}(x)}, \\
F_{A \cup B}(x) & =\min \left(r_{A}(x), r_{B}(x)\right) \cdot e^{i \omega_{A \cup B}(x)} .
\end{aligned}
$$

(ii) The intersection of $A$ and $B$, denoted as $A \cap_{N} B$, is defined as:

$$
A \cap_{N} B=\left\{\left(x, T_{A \cap B}(x), I_{A \cap B}(x), F_{A \cap B}(x)\right): x \in X\right\},
$$

where $T_{A \cap B}(x), I_{A \cap B}(x), F_{A \cap B}(x)$ are given by:

$$
\begin{aligned}
& T_{A \cap B}(x)=\min \left(p_{A}(x), p_{B}(x)\right) \cdot e^{i \mu_{A \cap B}(x)}, \\
& I_{A \cap B}(x)=\max \left(q_{A}(x), q_{B}(x)\right) \cdot e^{i v_{A \cap B}(x)}, \\
& F_{A \cap B}(x)=\max \left(r_{A}(x), r_{B}(x)\right) \cdot e^{i \omega_{A \cap B}(x)}
\end{aligned}
$$

The union and the intersection of the phase terms of the complex truth, falsity and indeterminacy membership functions can be calculated from, but not limited to, any one of the following operations: 
(a) Sum:

$$
\begin{gathered}
\mu_{A \cup B}(x)=\mu_{A}(x)+\mu_{B}(x), \\
v_{A \cup B}(x)=v_{A}(x)+v_{B}(x), \\
\omega_{A \cup B}(x)=\omega_{A}(x)+\omega_{B}(x) .
\end{gathered}
$$

(b) Max:

$$
\begin{aligned}
\mu_{A \cup B}(x) & =\max \left(\mu_{A}(x), \mu_{B}(x)\right), \\
v_{A \cup B}(x) & =\max \left(v_{A}(x), v_{B}(x)\right), \\
\omega_{A \cup B}(x) & =\max \left(\omega_{A}(x), \omega_{B}(x)\right) .
\end{aligned}
$$

(c) Min:

$$
\begin{aligned}
\mu_{A \cup B}(x) & =\min \left(\mu_{A}(x), \mu_{B}(x)\right), \\
v_{A \cup B}(x) & =\min \left(v_{A}(x), v_{B}(x)\right), \\
\omega_{A \cup B}(x) & =\min \left(\omega_{A}(x), \omega_{B}(x)\right) .
\end{aligned}
$$

(d) "The game of winner, neutral, and loser":

$$
\begin{aligned}
& \mu_{A \cup B}(x)=\left\{\begin{array}{lll}
\mu_{A}(x) & \text { if } & p_{A}>p_{B} \\
\mu_{B}(x) & \text { if } & p_{B}>p_{A}
\end{array},\right. \\
& v_{A \cup B}(x)=\left\{\begin{array}{lll}
v_{A}(x) & \text { if } & q_{A}<q_{B} \\
v_{B}(x) & \text { if } & q_{B}<q_{A}
\end{array},\right. \\
& \omega_{A \cup B}(x)=\left\{\begin{array}{lll}
\omega_{A}(x) & \text { if } & r_{A}<r_{B} \\
\omega_{B}(x) & \text { if } & r_{B}<r_{A}
\end{array} .\right.
\end{aligned}
$$

Definition 5 [17]. Let the following statements hold:

(a) $V$ is a non-void set.

(b) $\breve{\rho}_{T}, \breve{\rho}_{I}, \breve{\rho}_{F}$ are three functions, each from $V$ to $[0,1]$.

(c) $\breve{\omega}_{T}, \breve{\omega}_{I}, \breve{\omega}_{F}$ are three functions, each from $V \times V$ to $[0,1]$.

(d) $\breve{\rho}=\left(\breve{\rho}_{T}, \breve{\rho}_{I}, \breve{\rho}_{F}\right)$ and $\breve{\omega}=\left(\breve{\omega}_{T}, \breve{\omega}_{I}, \breve{\omega}_{F}\right)$.

Then the structure $\breve{\xi}=\langle V, \breve{\rho}, \breve{\omega}\rangle$ is said to be a generalized single valued neutrosophic graph of type 1 (GSVNG1).

\section{Remark 1.}

(i) $\breve{\rho}$ depends on $\breve{\rho}_{T}, \breve{\rho}_{I}, \breve{\rho}_{F}$ and $\breve{\omega}$ depends on $\breve{\omega}_{T}, \breve{\omega}_{I}, \breve{\omega}_{F}$. Hence there are seven mutually independent parameters in total that make up a CNG1: V, $\breve{\rho}_{T}, \breve{\rho}_{I}, \breve{\rho}_{F}, \breve{\omega}_{T}, \breve{\omega}_{I}, \breve{\omega}_{F}$.

(ii) For each $x \in V, x$ is said to be a vertex of $\breve{\xi}$. The entire set $V$ is thus called the vertex set of $\breve{\xi}$.

(iii) For each $u, v \in V,(u, v)$ is said to be a directed edge of $\breve{\xi}$. In particular, $(v, v)$ is said to be a loop of $\breve{\xi}$

(iv) For each vertex: $\breve{\rho}_{T}(v), \breve{\rho}_{I}(v), \breve{\rho}_{F}(v)$ are called the truth-membership value, indeterminate membership value, and false-membership value, respectively, of that vertex $v$. Moreover, if $\breve{\rho}_{T}(v)=\breve{\rho}_{I}(v)=$ $\breve{\rho}_{F}(v)=0$, then $v$ is said to be a void vertex.

(v) Likewise, for each edge $(u, v): \breve{\omega}_{T}(u, v), \breve{\omega}_{I}(u, v), \breve{\omega}_{F}(u, v)$ are called the truth-membership value, indeterminate-membership value, and false-membership value, respectively of that directed edge $(u, v)$. Moreover, if $\breve{\omega}_{T}(u, v)=\breve{\omega}_{I}(u, v)=\breve{\omega}_{F}(u, v)=0$, then $(u, v)$ is said to be a void directed edge.

Here we shall restate the concept of complex fuzzy graph of type 1 . Moreover, for all the remaining parts of this paper, we shall denote the set $\{z \in \mathbb{C}:|z| \leq 1\}$ as $O_{1}$.

Definition 6 [27]. Let the following statements hold: 
(a) $V$ is a non-void set.

(b) $\dot{\rho}$ is a function from $V$ to $O_{1}$.

(c) $\dot{\omega}$ is a function from $V \times V$ to $O_{1}$.

Then:

(i) the structure $\dot{\xi}=\langle V, \dot{\rho}, \dot{\omega}\rangle$ is said to be a complex fuzzy graph of type 1 (abbr. CFG1).

(ii) For each $x \in V, x$ is said to be a vertex of $\dot{\xi}$. The entire set $V$ is thus called the vertex set of $\dot{\xi}$.

(iii) For each $u, v \in V,(u, v)$ is said to be a directed edge of $\dot{\xi}$. In particular, $(v, v)$ is said to be a loop of $\dot{\xi}$.

\section{Complex Neutrosophic Graphs of Type 1}

By using the concept of complex neutrosophic sets [26], the concept of complex fuzzy graph of type 1 [27], and the concept of generalized single valued neutrosophic graph of type 1 [17], we define the concept of complex neutrosophic graph of type 1 as follows:

Definition 7. Let the following statements hold:

(a) $\quad V$ is a non-void set.

(b) $\rho_{T}, \rho_{I}, \rho_{F}$ are three functions, each from $V$ to $O_{1}$.

(c) $\omega_{T}, \omega_{I}, \omega_{F}$ are three functions, each from $V \times V$ to $O_{1}$.

(d) $\rho=\left(\rho_{T}, \rho_{I}, \rho_{F}\right)$ and $\omega=\left(\omega_{T}, \omega_{I}, \omega_{F}\right)$.

Then the structure $\xi=\langle V, \rho, \omega\rangle$ is said to be a complex neutrosophic graph of type 1 (abbr. CNG1).

Remark 2. $\rho$ depends on $\rho_{T}, \rho_{I}, \rho_{F}$, and $\omega$ depends on $\omega_{T}, \omega_{I}, \omega_{F}$. Hence there are seven mutually independent parameters in total that make up a CNG1: $V, \rho_{T}, \rho_{I}, \rho_{F}, \omega_{T}, \omega_{I}, \omega_{F}$. Furthermore, in analogy to a GSVNG1:

(i) For each $x \in V, x$ is said to be a vertex of $\xi$. The entire set $V$ is thus called the vertex set of $\xi$.

(ii) For each $u, v \in V,(u, v)$ is said to be a directed edge of $\xi$. In particular, $(v, v)$ is said to be a loop of $\xi$.

(iii) For each vertex: $\rho_{T}(v), \rho_{I}(v), \rho_{F}(v)$ are called the complex truth, indeterminate, and falsity membership values, respectively, of the vertex $v$. Moreover, if $\rho_{T}(v)=\rho_{I}(v)=\rho_{F}(v)=0$, then $v$ is said to be a void vertex.

(iv) Likewise, for each directed edge $(u, v): \omega_{T}(u, v), \omega_{I}(u, v), \omega_{F}(u, v)$ are called the complex truth, indeterminate and falsity membership value, of the directed edge $(u, v)$. Moreover, if $\omega_{T}(u, v)=$ $\omega_{I}(u, v)=\omega_{F}(u, v)=0$, then $(u, v)$ is said to be a void directed edge.

For the sake of brevity, we shall denote $\omega(u, v)=\left(\omega_{T}(u, v), \omega_{I}(u, v), \omega_{F}(u, v)\right)$ and $\rho(v)=$ $\left(\rho_{T}(v), \rho_{I}(v), \rho_{F}(v)\right)$ for all the remaining parts of this paper.

As mentioned, CNG1 is generalized from both GSVNG1 and CFG1. As a result, we have $\omega_{T}, \omega_{I}$ and $\omega_{T}$ being functions themselves. This further implies that $\omega_{T}(u, v), \omega_{I}(u, v)$ and $\omega_{T}(u, v)$ can only be single values from $\mathrm{O}_{1}$. In particular, $\omega_{T}(v, v), \omega_{I}(v, v)$, and $\omega_{T}(v, v)$ can only be single values.

As a result, each vertex $v$ in a CNG1 possess a single, undirected loop, whether void or not. And each of the two distinct vertices $u, v$ in a CNG1 possess two directed edges, resulting from $(u, v)$ and $(v, u)$, whether void or not.

Recall that in classical graph theory, we often deal with ordinary (or undirected) graphs, and also simple graphs. To further relate our CNG1 with it, we now proceed with the following definition.

Definition 8. Let $\xi=\langle V, \rho, \omega\rangle$ be a CNG1.

(a) If $\omega(a, b)=\omega(b, a)$, then $\{a, b\}=\{(a, b),(b, a)\}$ is said to be an (ordinary) edge of $\xi$. Moreover, $\{a, b\}$ is said to be a void (ordinary) edge if both $(a, b)$ and $(b, a)$ are void. 
(b) If $\omega(u, v)=\omega(v, u)$ holds for all $u, v \in V$, then $\xi$ is said to be ordinary (or undirected), otherwise it is said to be directed.

(c) If all the loops of $\xi$ are void, then $\xi$ is said to be simple.

Definition 9. Let $\xi=\langle V, \rho, \omega\rangle$ be an ordinary $C N G 1$. If for all $u, v \in V$ with $u \neq v$, there exist non-void edges $\left\{u=w_{1}, w_{2}\right\},\left\{w_{2}, w_{3}\right\}, \ldots,\left\{w_{n-1}, w_{n}=v\right\}$ for some $n \geq 2$, then $\xi$ is said to be connected.

Definition 10. Let $\xi=\langle V, \rho, \omega\rangle$ be an ordinary CNG1. Let $u, v \in V$. Then:

(a) $\{u, v\}$ is said to be adjacent to $u$ (and to $v$ ).

(b) $u$ (and $v$ as well) is said to be an end-point of $\{u, v\}$.

We now discuss a real life scenario that can only be represented by a CNG1.

\subsection{The Scenario}

Note: All the locations mentioned are fictional

Suppose there is a residential area in Malaysia with four families: $a, b, c, d$. All of them have Internet access. In other words, they are Internet clients, which will access the Internet servers from around the world (including those servers located within Malaysia) depending on which website they are visiting.

If they access the internet on their own, the outcomes can be summarized as given in the Table 1 and Figure 1.

Table 1. The outcomes of individuals, for Scenario 3.1.

\begin{tabular}{|c|c|c|c|c|}
\hline Activities & $a$ & $b$ & $c$ & $d$ \\
\hline $\begin{array}{l}\text { Some members will } \\
\text { seek excitement } \\
\text { (e.g., playing } \\
\text { online games) }\end{array}$ & $\begin{array}{l}\text { Happens on } 80 \% \text { of the day, and } \\
\text { those will be connecting } \\
\text { towards } 0^{\circ} \text { (because that server } \\
\text { is located in China } * \text { ) }\end{array}$ & $\begin{array}{l}\text { Happens on } 70 \% \text { of } \\
\text { the day, and those } \\
\text { will be connecting } \\
\text { towards } 30^{\circ}\end{array}$ & $\begin{array}{l}\text { Happens on } 90 \% \text { of } \\
\text { the day, and those } \\
\text { will be connecting } \\
\text { towards } 120^{\circ}\end{array}$ & $\begin{array}{l}\text { Happens on } 80 \% \text { of } \\
\text { the day, and those } \\
\text { will be connecting } \\
\text { towards } 250^{\circ}\end{array}$ \\
\hline $\begin{array}{l}\text { Some members will } \\
\text { want to surf around } \\
\text { (e.g., } \\
\text { online shopping) }\end{array}$ & $\begin{array}{l}\text { Happens on } 50 \% \text { of the day, and } \\
\text { those will be connecting } \\
\text { towards } 130^{\circ} \text { (because that } \\
\text { server is located in Australia } * \text { ) }\end{array}$ & $\begin{array}{l}\text { Happens on } 60 \% \text { of } \\
\text { the day, and those } \\
\text { will be connecting } \\
\text { towards } 180^{\circ}\end{array}$ & $\begin{array}{l}\text { Happens on } 20 \% \text { of } \\
\text { the day, and those } \\
\text { will be connecting } \\
\text { towards } 340^{\circ}\end{array}$ & $\begin{array}{l}\text { Happens on } 40 \% \text { of } \\
\text { the day, and those } \\
\text { will be connecting } \\
\text { towards } 200^{\circ}\end{array}$ \\
\hline $\begin{array}{l}\text { Some members will } \\
\text { need to relax } \\
\text { (e.g., listening } \\
\text { to music) }\end{array}$ & $\begin{array}{l}\text { Happens on } 20 \% \text { of the day, and } \\
\text { those will be connecting } \\
\text { towards } 220^{\circ} \text { (because that } \\
\text { server is located in Sumatra } \\
\text { *, Indonesia) }\end{array}$ & $\begin{array}{l}\text { Happens on } 30 \% \text { of } \\
\text { the day, and those } \\
\text { will be connecting } \\
\text { towards } 200^{\circ}\end{array}$ & $\begin{array}{l}\text { Happens on } 50 \% \text { of } \\
\text { the day, and those } \\
\text { will be connecting } \\
\text { towards } 40^{\circ}\end{array}$ & $\begin{array}{l}\text { Happens on } 10 \% \text { of } \\
\text { the day, and those } \\
\text { will be connecting } \\
\text { towards } 110^{\circ}\end{array}$ \\
\hline
\end{tabular}

(*) as illustrated in Figure 1.

Moreover, the following (unordered) pairs of the four families are close friends or relatives:

$$
\{a, b\},\{a, c\},\{a, d\},\{b, d\} \text {. }
$$

Thus, each pair of family mentioned (e.g., $\{a, b\})$ may invite one another for a visit, accessing the Internet as one team. In particular:

(i) When $\{a, b\}$ or $\{a, d\}$ access the internet together, they will simply search for "a place of common interest". This is regardless of who initiates the invitation.

(ii) $\quad a$ and $c$ rarely meet. Thus, each time they do, everyone (especially the children) will be so excited that they would like to try something fresh, so all will seek excitement and connect towards to a local broadcasting server at $240^{\circ}$ to watch soccer matches (that server will take care of which country to connect to) for the entire day. This is also regardless of who initiates the visitation. 
(iii) The size and the wealth of $d$ far surpasses $b$. Thus, it would always be $d$ who invites $b$ to their house, never the other way, and during the entire visit, members of $b$ will completely behave like members of $d$ and, therefore, will visit the same websites as $d$.

Denote the first term of the ordered pair $(u, v)$ as the family who initiates the invitation, and the second term as family who receives the invitation and visit the other family. The outcomes of the seven possible teams $(a, b),(a, c),(a, d),(b, a),(c, a),(d, a),(d, b)$ are, thus, summarized by Table 2.

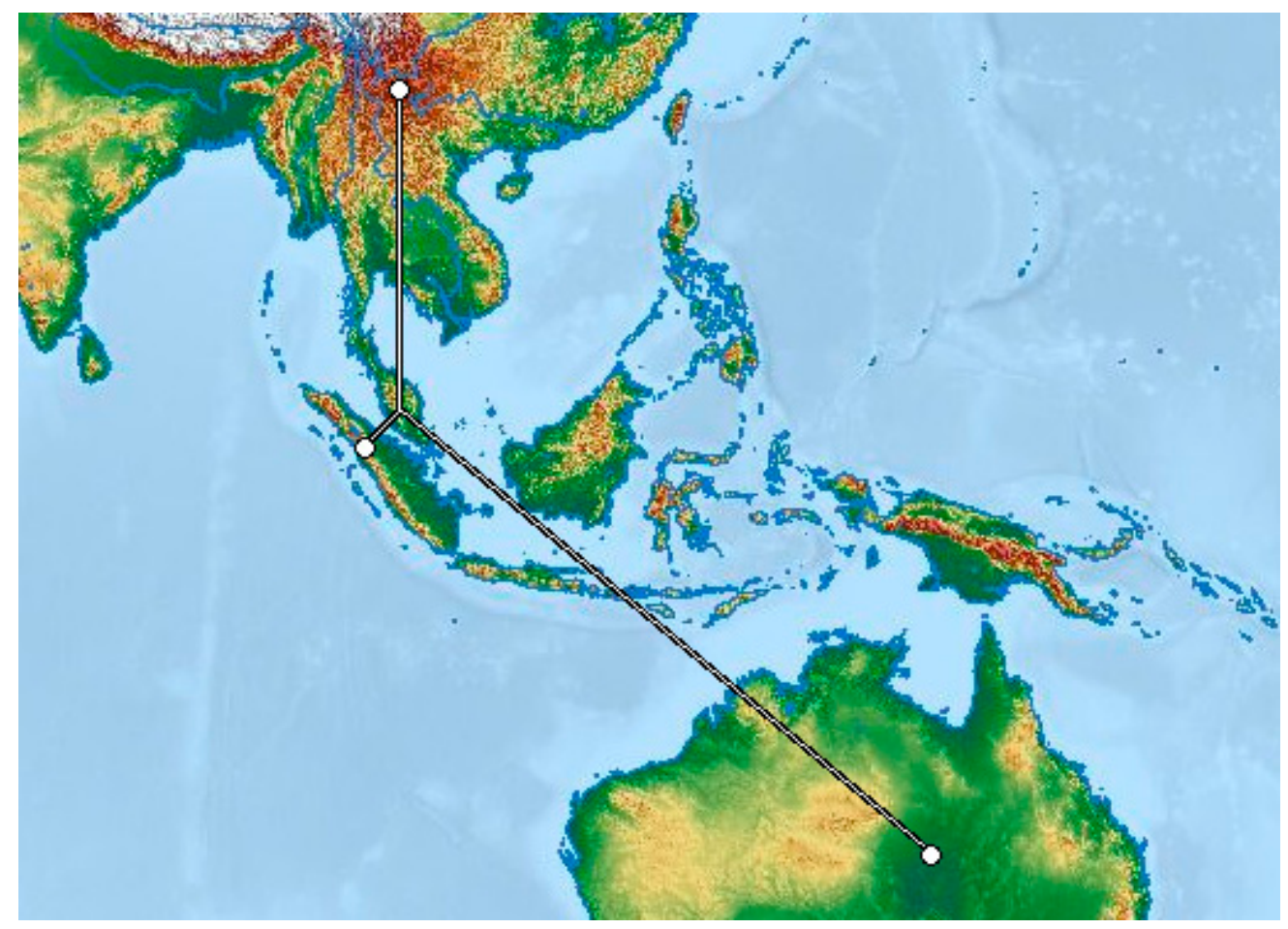

Figure 1. The illustration of the servers' relative positions using a public domain map, for Scenario 3.1.

Table 2. The outcomes of teams in pairs, for the scenario.

\begin{tabular}{ccccc}
\hline Activities & $(\boldsymbol{a}, \boldsymbol{b}),(\boldsymbol{b}, \boldsymbol{a})$ & $(\boldsymbol{a}, \boldsymbol{c}),(\boldsymbol{c}, \boldsymbol{a})$ & $(\boldsymbol{a}, \boldsymbol{d}),(\boldsymbol{d}, \boldsymbol{a})$ & $(\boldsymbol{d}, \boldsymbol{b})$ \\
\hline $\begin{array}{c}\text { Some members will } \\
\text { seek excitement }\end{array}$ & $\begin{array}{c}\text { Happens on } 80 \% \text { of } \\
\text { the day, and those } \\
\text { will be connecting } \\
\text { towards } 15^{\circ}\end{array}$ & $\begin{array}{c}\text { Happens on the } \\
\text { entire day, all will } \\
\text { be connecting } \\
\text { towards } 240^{\circ}\end{array}$ & $\begin{array}{c}\text { Happens on } 80 \% \text { of } \\
\text { the day, and those } \\
\text { will be connecting } \\
\text { towards } 305^{\circ}\end{array}$ & $\begin{array}{c}\text { Happens on } 80^{\circ} \text { of } \\
\text { the day, and those } \\
\text { will be connecting } \\
\text { towards } 250^{\circ}\end{array}$ \\
\hline $\begin{array}{c}\text { Some members } \\
\text { will want to surf } \\
\text { around }\end{array}$ & $\begin{array}{c}\text { Happens on } 60 \% \text { of } \\
\text { the day, and those } \\
\text { will be connecting } \\
\text { towards } 155^{\circ}\end{array}$ & Does not happen & $\begin{array}{c}\text { Happens on } 50 \% \text { of } \\
\text { the day, and those } \\
\text { will be connecting } \\
\text { towards } 165^{\circ}\end{array}$ & $\begin{array}{c}\text { Happens on } 40 \% \text { of } \\
\text { the day, and those } \\
\text { will be connecting } \\
\text { towards } 200^{\circ}\end{array}$ \\
\hline $\begin{array}{c}\text { Some members } \\
\text { will need to relax }\end{array}$ & $\begin{array}{c}\text { Happens on } 30^{\circ} \text { of } \\
\text { the day, and those } \\
\text { will be connecting } \\
\text { towards } 210^{\circ}\end{array}$ & Does not happen & $\begin{array}{c}\text { Happens on } 50^{\circ} \text { of } \\
\text { the day, and those } \\
\text { will be connecting } \\
\text { towards } 40^{\circ}\end{array}$ & $\begin{array}{c}\text { Happens on } 10^{\circ} \text { of } \\
\text { the day, and those } \\
\text { will be connecting } \\
\text { towards } 110^{\circ}\end{array}$ \\
\hline
\end{tabular}

On the other hand, $\{c, b\}$ and $\{d, c\}$ are mutual strangers. So $c$ and $b$ will visit each other. The same goes to $d$ and $c$. 


\subsection{Representation of the Scenario with CNG1}

We now follow all the steps from (a) to (e) in Definition 7, to represent the scenario with a particular CNG1.

(a) Take $V_{0}=\{a, b, c, d\}$.

(b) In accordance with the scenario, define the three functions on $V_{0}: \rho_{T}, \rho_{I}, \rho_{F}$, as illustrated in Table 3.

Table 3. $k(v)$, where $k$ represents any of the 3 functions on $V_{0} \rho_{T}, \rho_{I}, \rho_{F}$, for the scenario. Also mentioned in Section 4.2.

\begin{tabular}{ccccc}
\hline $\boldsymbol{k}$ & $\boldsymbol{v}$ & $\boldsymbol{a}$ & $\boldsymbol{c}$ & $\boldsymbol{d}$ \\
\hline$\rho_{T}$ & $0.8 \mathrm{e}^{i 2 \pi}$ & $0.7 \mathrm{e}^{i \frac{\pi}{6}}$ & $0.9 \mathrm{e}^{i \frac{2 \pi}{3}}$ & $0.8 \mathrm{e}^{i \frac{25 \pi}{18}}$ \\
$\rho_{I}$ & $0.5 \mathrm{e}^{i \frac{13 \pi}{18}}$ & $0.6 \mathrm{e}^{i \pi}$ & $0.2 \mathrm{e}^{i \frac{17 \pi}{9}}$ & $0.4 \mathrm{e}^{i \frac{10 \pi}{9}}$ \\
$\rho_{F}$ & $0.2 \mathrm{e}^{i \frac{11 \pi}{9}}$ & $0.3 \mathrm{e}^{i \frac{10 \pi}{9}}$ & $0.5 \mathrm{e}^{i \frac{2 \pi}{9}}$ & $0.1 \mathrm{e}^{i \frac{11 \pi}{18}}$ \\
\hline
\end{tabular}

(c) In accordance with the scenario, define the three functions $\omega_{T}, \omega_{I}, \omega_{F}$, as illustrated in Tables 4-6.

Table 4. The outcomes of $\omega_{T}(u, v)$, for the scenario. Also mentioned in Section 4.2.

\begin{tabular}{|c|c|c|c|c|}
\hline$u^{v}$ & $a$ & $b$ & $c$ & $d$ \\
\hline$a$ & 0 & $0.8 \mathrm{e}^{i \frac{\pi}{12}}$ & $1 \mathrm{e}^{i \frac{4 \pi}{3}}$ & $0.8 \mathrm{e}^{i \frac{61 \pi}{36}}$ \\
\hline$b$ & $0.8 \mathrm{e}^{i \frac{\pi}{12}}$ & 0 & 0 & 0 \\
\hline$c$ & $1 \mathrm{e}^{i \frac{4 \pi}{3}}$ & 0 & 0 & 0 \\
\hline$d$ & $0.8 \mathrm{e}^{i \frac{61 \pi}{36}}$ & $0.8 \mathrm{e}^{i \frac{25 \pi}{18}}$ & 0 & 0 \\
\hline
\end{tabular}

Table 5. The outcomes of $\omega_{I}(u, v)$, for the scenario. Also mentioned in Section 4.2.

\begin{tabular}{cccccc}
\hline $\boldsymbol{u}$ & $\boldsymbol{v}$ & $\boldsymbol{a}$ & $\boldsymbol{b}$ & $\boldsymbol{c}$ & $\boldsymbol{d}$ \\
\hline$a$ & 0 & $0.6 \mathrm{e}^{i \frac{31 \pi}{36}}$ & 0 & $0.5 \mathrm{e}^{i \frac{33 \pi}{36}}$ \\
$b$ & $0.6 \mathrm{e}^{i \frac{31 \pi}{36}}$ & 0 & 0 & 0 \\
$c$ & 0 & 0 & 0 & 0 \\
$d$ & $0.5 \mathrm{e}^{i \frac{33 \pi}{36}}$ & $0.4 \mathrm{e}^{i \frac{10 \pi}{9}}$ & 0 & 0 \\
\hline
\end{tabular}

Table 6. The outcomes of $\omega_{F}(u, v)$, for the scenario. Also mentioned in Section 4.2.

\begin{tabular}{ccccc}
\hline $\boldsymbol{u}$ & $\boldsymbol{a}$ & $\boldsymbol{b}$ & $\boldsymbol{c}$ & $\boldsymbol{d}$ \\
\hline$a$ & 0 & $0.3 \mathrm{e}^{i \frac{7 \pi}{6}}$ & 0 & $0.5 \mathrm{e}^{i \frac{2 \pi}{9}}$ \\
$b$ & $0.3 \mathrm{e}^{i \frac{7 \pi}{6}}$ & 0 & 0 & 0 \\
$c$ & 0 & 0 & 0 & 0 \\
$d$ & $0.5 \mathrm{e}^{i \frac{2 \pi}{9}}$ & $0.1 \mathrm{e}^{i \frac{11 \pi}{18}}$ & 0 & 0 \\
\hline
\end{tabular}

(d) By statement (d) from Definition 7, let $\rho_{0}=\left(\rho_{T}, \rho_{I}, \rho_{F}\right)$, and $\omega_{0}=\left(\omega_{T}, \omega_{I}, \omega_{F}\right)$. We have now formed a $\mathrm{CNG} 1\left\langle V_{0}, \rho_{0}, \omega_{0}\right\rangle$.

One of the way of representing the entire $\left\langle V_{0}, \rho_{0}, \omega_{0}\right\rangle$ is by using a diagram that is analogous with graphs as in classical graph theory, as shown in the Figure 2. 


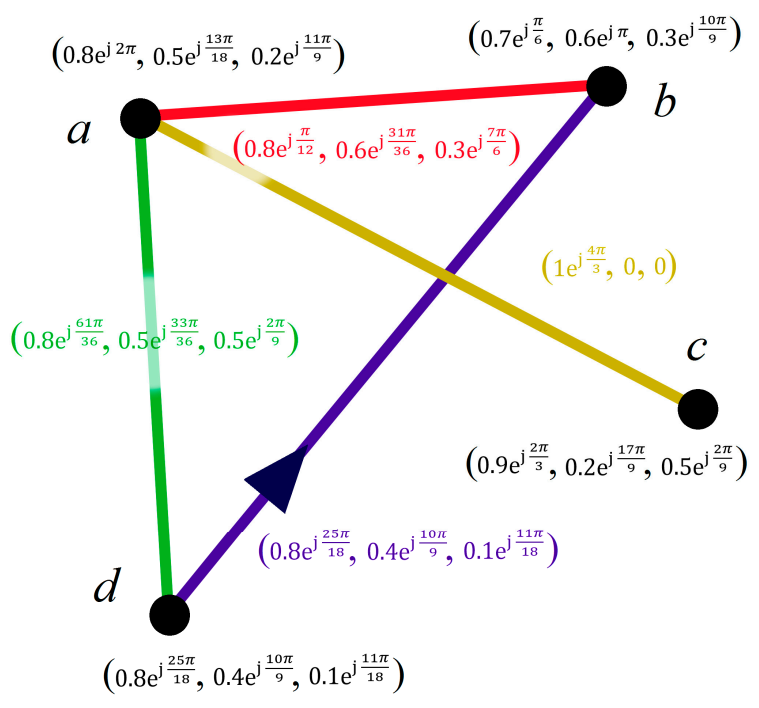

Figure 2. A diagram representing $\left\langle V_{0}, \rho_{0}, \omega_{0}\right\rangle$, for the scenario.

In other words, only the non-void edges (whether directed or ordinary) and vertices are to be drawn in such a diagram.

Hence, we have shown how a CGN1 can be constructed from a data set with four homes. The same concept mentioned can certainly be used on a larger dataset, such as one with thousands of locations and thousands of homes, which will result in a more complicated diagram being generated. However, one will definitely require computer algebraic systems, such as SAGE, to process the data and to display the data in diagram form.

Additionally, recall that, in classical graph theory, a graph can be represented by an adjacency matrix, for which the entries are either a positive integer (connected) or 0 (not connected).

This motivates us to represent CNG1 using a matrix as well, in a similar manner. Nonetheless, instead of a single value that is either 0 or 1 , we have three values to deal with: $\omega_{T}, \omega_{I}, \omega_{F}$, with each of them capable of being anywhere in $O_{1}$. Moreover, each of the vertices themselves also contain $\rho_{T}$, $\rho_{I}, \rho_{F}$, which must be taken into account as well.

\section{Representation of a CNG1 by an Adjacency Matrix}

\subsection{Two Methods of Representation}

In this section, we discuss the representation of CNG1 in two ways, which are both analogous to the one encountered in classical literature.

Let $\xi=\langle V, \rho, \omega\rangle$ be a CNG1 where vertex set $V=\left\{v_{1}, v_{2}, \ldots, v_{n}\right\}$ (i.e., CNG1 has finite vertices). We first form an $n \times n$ matrix as shown:

$$
\mathbf{M}=\left[\mathbf{a}_{i, j}\right]_{n}=\left(\begin{array}{cccc}
\mathbf{a}_{1,1} & \mathbf{a}_{1,2} & \ldots & \mathbf{a}_{1, n} \\
\mathbf{a}_{2,1} & \mathbf{a}_{2,2} & & \mathbf{a}_{2, n} \\
\vdots & & \ddots & \vdots \\
\mathbf{a}_{n, 1} & \mathbf{a}_{n, 2} & \cdots & \mathbf{a}_{n, n}
\end{array}\right),
$$

where $\mathbf{a}_{i, j}=\omega\left(v_{i}, v_{j}\right)$ for all $i, j$.

In other words, each element of the matrix $\mathbf{M}$ is itself an ordered set of three elements, instead of just a number of either 0 or 1 in the classical literature.

Remark 3. Since $\xi$ can only possess undirected loops, we decided not to multiply the main diagonal elements of $\mathbf{M}$ by 2, as seen in adjacency matrices for graphs classical literature (2 for undirected, 1 for directed, 0 for void). 
Meanwhile, also recall that each of the vertices in $\xi$ contains $\rho_{T}, \rho_{I}, \rho_{F}$, which must be taken into account as well.

Thus, we form another matrix $\mathbf{K}$ as shown:

$$
\mathbf{K}=\left[\mathbf{k}_{i}\right]_{n, 1}=\left(\begin{array}{c}
\mathbf{k}_{1} \\
\mathbf{k}_{2} \\
\vdots \\
\mathbf{k}_{n}
\end{array}\right) \text {, where } \mathbf{k}_{i}=\rho\left(v_{i}\right) \text { foralli } .
$$

To accomplish one of our methods of representing the entire $\xi$ we, therefore, augment the matrix $\mathbf{K}$ with $\mathbf{M}$, forming the adjacency matrix of $\mathrm{CNG1}$, [K| M], as shown:

$$
[\mathbf{K} \mid \mathbf{M}]=\left(\begin{array}{ccccc}
\mathbf{k}_{1} & \mathbf{a}_{1,1} & \mathbf{a}_{1,2} & \ldots & \mathbf{a}_{1, n} \\
\mathbf{k}_{2} & \mathbf{a}_{2,1} & \mathbf{a}_{2,2} & & \mathbf{a}_{2, n} \\
& \vdots & & \ddots & \vdots \\
\mathbf{k}_{n} & \mathbf{a}_{n, 1} & \mathbf{a}_{n, 2} & \cdots & \mathbf{a}_{n, n}
\end{array}\right),
$$

where $\mathbf{a}_{i, j}=\omega\left(v_{i}, v_{j}\right)$, and $\mathbf{k}_{i}=\rho\left(v_{i}\right)$, for all $i, j$.

Although $[\mathbf{K} \mid \mathbf{M}]$ is an $n \times(n+1)$ matrix and therefore not a square, this representation will save us another separate ordered set to represent the $\rho_{T}, \rho_{I}, \rho_{F}$ values of the vertices themselves.

Sometimes it is more convenient to separately deal with each of the three kinds of membership values for both edges and vertices. As a result, here we provide another method of representing the entire $\xi$ : using three $n \times(n+1)$ matrices, $[\mathbf{K} \mid \mathbf{M}]_{T},[\mathbf{K} \mid \mathbf{M}]_{I}$, and $[\mathbf{K} \mid \mathbf{M}]_{F}$, each derived from $[\mathbf{K} \mid \mathbf{M}]$ by taking only one kind of the membership values from its elements:

$$
[\mathbf{K} \mid \mathbf{M}]_{T}=\left[\mathbf{K}_{T} \mid \mathbf{M}_{T}\right]\left(\begin{array}{ccccc}
\rho_{T}\left(v_{1}\right) & \omega_{T}\left(v_{1}, v_{1}\right) & \omega_{T}\left(v_{1}, v_{2}\right) & \ldots & \omega_{T}\left(v_{1}, v_{n}\right) \\
\rho_{T}\left(v_{2}\right) & \omega_{T}\left(v_{2}, v_{1}\right) & \omega_{T}\left(v_{2}, v_{2}\right) & \cdots & \omega_{T}\left(v_{2}, v_{n}\right) \\
& \vdots & & \ddots & \vdots \\
\rho_{T}\left(v_{n}\right) & \omega_{T}\left(v_{n}, v_{1}\right) & \omega_{T}\left(v_{n}, v_{2}\right) & \ldots & \omega_{T}\left(v_{n}, v_{n}\right)
\end{array}\right),
$$

$[\mathbf{K} \mid \mathbf{M}]_{T},[\mathbf{K} \mid \mathbf{M}]_{I}$, and $[\mathbf{K} \mid \mathbf{M}]_{F}$ shall, thus, be called, respectively, the truth-adjacency matrix, the indeterminate-adjacency matrix, and the false-adjacency matrix of $\xi$.

Remark 4. If $[\mathbf{K} \mid \mathbf{M}]_{I}=[\mathbf{K} \mid \mathbf{M}]_{F}=[0]_{n, n+1}, \mathbf{K}_{T}=[1]_{n, 1}$, all the entries of $\mathbf{M}_{T}$ are either 1 or 0 , then $\xi$ is reduced to a graph in classical literature. Furthermore, if that $\mathbf{M}_{T}$ is symmetrical and with main diagonal elements being zero, then $\xi$ is further reduced to a simple ordinary graph in the classical literature.

Remark 5. If $[\mathbf{K} \mid \mathbf{M}]_{I}=[\mathbf{K} \mid \mathbf{M}]_{F}=[0]_{n, n+1}$, and all the entries of $[\mathbf{K} \mid \mathbf{M}]_{T}$ are real values from the interval $[0,1]$, then $\xi$ is reduced to a generalized fuzzy graph type 1 (GFG1).

Remark 6. If all the entries of $[\mathbf{K} \mid \mathbf{M}]_{T},[\mathbf{K} \mid \mathbf{M}]_{I}$, and $[\mathbf{K} \mid \mathbf{M}]_{F}$ are real values from the interval $[0,1]$, then $\xi$ is reduced to a generalized single valued neutrosophic graphs of type 1 (GSVNG1). 
Remark 7. If $\mathbf{M}_{T}, \mathbf{M}_{I}$, and $\mathbf{M}_{F}$ are symmetric matrices, then $\xi$ is ordinary.

\subsection{Illustrative Example}

For the sake of brevity, we now give representation for our example for the scenario in 3.1 by the latter method using three matrices: $[\mathbf{K} \mid \mathbf{M}]_{T},[\mathbf{K} \mid \mathbf{M}]_{I}$, and $[\mathbf{K} \mid \mathbf{M}]_{F}$ :

$$
\begin{aligned}
& {[\mathbf{K} \mid \mathbf{M}]_{T}=\left(\begin{array}{ccccc}
0.8 \mathrm{e}^{i 2 \pi} & 0 & 0.8 \mathrm{e}^{i \frac{\pi}{12}} & 1 \mathrm{e}^{i \frac{4 \pi}{3}} & 0.8 \mathrm{e}^{i \frac{61 \pi}{36}} \\
0.7 \mathrm{e}^{i \frac{\pi}{6}} & 0.8 \mathrm{e}^{i \frac{\pi}{12}} & 0 & 0 & 0 \\
0.9 \mathrm{e}^{i \frac{2 \pi}{3}} & 1 \mathrm{e}^{i \frac{4 \pi}{3}} & 0 & 0 & 0 \\
0.8 \mathrm{e}^{i \frac{25 \pi}{18}} & 0.8 \mathrm{e}^{i \frac{61 \pi}{36}} & 0.8 \mathrm{e}^{i \frac{25 \pi}{18}} & 0 & 0
\end{array}\right)} \\
& {[\mathbf{K} \mid \mathbf{M}]_{I}=\left(\begin{array}{ccccc}
0.5 \mathrm{e}^{i \frac{13 \pi}{18}} & 0 & 0.6 \mathrm{e}^{i \frac{31 \pi}{36}} & 0 & 0.5 \mathrm{e}^{i \frac{33 \pi}{36}} \\
0.6 \mathrm{e}^{i \pi} & 0.6 \mathrm{e}^{i \frac{31 \pi}{36}} & 0 & 0 & 0 \\
0.2 \mathrm{e}^{i \frac{17 \pi}{9}} & 0 & 0 & 0 & 0 \\
0.4 \mathrm{e}^{i \frac{10 \pi}{9}} & 0.5 \mathrm{e}^{i \frac{33 \pi}{36}} & 0.4 \mathrm{e}^{i \frac{10 \pi}{9}} & 0 & 0
\end{array}\right)} \\
& {[\mathbf{K} \mid \mathbf{M}]_{F}=\left(\begin{array}{ccccc}
0.2 \mathrm{e}^{i \frac{11 \pi}{9}} & 0 & 0.3 \mathrm{e}^{i \frac{7 \pi}{6}} & 0 & 0.5 \mathrm{e}^{i \frac{2 \pi}{9}} \\
0.3 \mathrm{e}^{i \frac{10 \pi}{9}} & 0.3 \mathrm{e}^{i \frac{7 \pi}{6}} & 0 & 0 & 0 \\
0.5 \mathrm{e}^{i \frac{2 \pi}{9}} & 0 & 0 & 0 & 0 \\
0.1 \mathrm{e}^{i \frac{11 \pi}{18}} & 0.5 \mathrm{e}^{i \frac{2 \pi}{9}} & 0.1 \mathrm{e}^{i \frac{11 \pi}{18}} & 0 & 0
\end{array}\right)}
\end{aligned}
$$

As in Section 3, we have shown how a matrix representation of a CNG1 with $|V|=4$ can be constructed. Likewise, the same concept mentioned can certainly be used on a larger CNG1 but, again, one will definitely require computer algebraic systems, such as SAGE to process the data and to display such a matrix representation.

\section{Some Theoretical Results on Ordinary CNG1}

We now discuss some theoretical results that follows from the definition of ordinary CNG1, as well as its representation with adjacency matrix. Since we are concerned about ordinary CNG1, all the edges that we will be referring to are ordinary edges.

Definition 11. Let $\xi=\langle V, \rho, \omega\rangle$ be an ordinary CNG1. Let $V=\left\{v_{1}, v_{2}, \ldots, v_{n}\right\}$ be the vertex set of $\xi$. Then, for each $i$, the resultant degree of $v_{i}$, denoted as $D\left(v_{i}\right)$, is defined to be the ordered set $\left(D_{T}\left(v_{i}\right), D_{I}\left(v_{i}\right), D_{F}\left(v_{i}\right)\right)$, for which:

(a) $D_{T}\left(v_{i}\right)=\sum_{r=1}^{n} \omega_{T}\left(v_{i}, v_{r}\right)+\omega_{T}\left(v_{i}, v_{i}\right)$,

(b) $D_{I}\left(v_{i}\right)=\sum_{r=1}^{n} \omega_{I}\left(v_{i}, v_{r}\right)+\omega_{I}\left(v_{i}, v_{i}\right)$,

(c) $D_{F}\left(v_{i}\right)=\sum_{r=1}^{n} \omega_{F}\left(v_{i}, v_{r}\right)+\omega_{F}\left(v_{i}, v_{i}\right)$.

Remark 8. In analogy to classical graph theory, each undirected loop has both its ends connected to the same vertex, so is counted twice.

Remark 9. Each of the values of $D_{T}\left(v_{i}\right), D_{I}\left(v_{i}\right)$, and $D_{F}\left(v_{i}\right)$ need not be an integer as in a classical graph.

Definition 12. Let $\xi=\langle V, \rho, \omega\rangle$ be an ordinary CNG1. Let $V=\left\{v_{1}, v_{2}, \ldots, v_{n}\right\}$ be the vertex set of $\xi$. Then, the resultant amount of edges of $\xi$, denoted as $E_{\xi}$, is defined to be the ordered set $\left(E_{T}, E_{I}, E_{F}\right)$ for which:

(a) $\quad E_{T}=\sum_{\{r, s\} \subseteq\{1,2, \ldots, n\}} \omega_{T}\left(v_{r}, v_{s}\right)$, 
(b) $E_{I}=\sum_{\{r, s\} \subseteq\{1,2, \ldots, n\}} \omega_{I}\left(v_{r}, v_{s}\right)$,

(c) $E_{F}=\sum_{\{r, s\} \subseteq\{1,2, \ldots, n\}} \omega_{F}\left(v_{r}, v_{s}\right)$.

Remark 10. As in classical graph theory, each edge is counted only once, as shown by $\{r, s\} \subseteq\{1,2, \ldots, n\}$ in the expression. For example, if $\omega_{T}\left(v_{a}, v_{b}\right)$ is added, we will not add $\omega_{T}\left(v_{b}, v_{a}\right)$ again since $\{a, b\}=\{b, a\}$.

Remark 11. Each of the values of $E_{T}, E_{I}$ and $E_{F}$ need not be an integer as in a classical graph. As a result, we call it the "amount" of edges, instead of the "number" of edges as in the classical literature.

For each vertex $v_{i}$, just because $D\left(v_{i}\right)$ equals 0 , that does not mean that all the edges connect to $v_{i}$ are void. It could be two distinct edges $\left\{v_{i}, v_{1}\right\}$ and $\left\{v_{i}, v_{2}\right\}$ with $\omega_{T}\left(v_{i}, v_{1}\right)=-\omega_{T}\left(v_{i}, v_{2}\right)$, $\omega_{I}\left(v_{i}, v_{1}\right)=-\omega_{I}\left(v_{i}, v_{2}\right)$ and $\omega_{F}\left(v_{i}, v_{1}\right)=-\omega_{F}\left(v_{i}, v_{2}\right)$ (i.e., equal in magnitude, but opposite in phase). The same goes to the value of $E_{\xi}$. This differs from the classical theory of graphs and, therefore, it motivates us to look at a CNG1 in yet another approach. We, thus, further define the following:

Definition 13. Let $\xi=\langle V, \rho, \omega\rangle$ be an ordinary CNG1. Let $V=\left\{v_{1}, v_{2}, \ldots, v_{n}\right\}$ be the vertex set of $\xi$. Then, for each $i$, the absolute degree of $v_{i}$, denoted as $|D|\left(v_{i}\right)$, is defined to be the ordered set $\left(|D|_{T}\left(v_{i}\right),|D|_{I}\left(v_{i}\right),|D|_{F}\left(v_{i}\right)\right)$, for which:

(a) $|D|_{T}\left(v_{i}\right)=\sum_{r=1}^{n}\left|\omega_{T}\left(v_{i}, v_{r}\right)\right|+\left|\omega_{T}\left(v_{i}, v_{i}\right)\right|$,

(b) $|D|_{I}\left(v_{i}\right)=\sum_{r=1}^{n}\left|\omega_{I}\left(v_{i}, v_{r}\right)\right|+\left|\omega_{I}\left(v_{i}, v_{i}\right)\right|$,

(c) $|D|_{F}\left(v_{i}\right)=\sum_{r=1}^{n}\left|\omega_{F}\left(v_{i}, v_{r}\right)\right|+\left|\omega_{F}\left(v_{i}, v_{i}\right)\right|$.

Definition 14. Let $\xi=\langle V, \rho, \omega\rangle$ be an ordinary CNG1. Let $V=\left\{v_{1}, v_{2}, \ldots, v_{n}\right\}$ be the vertex set of $\xi$. Then, the absolute amount of edges of $\xi$, denoted as $|E|_{\xi}$, is defined to be the ordered set $\left(|E|_{T},|E|_{I^{\prime}}|E|_{F}\right)$ for which:

(a) $|E|_{T}=\sum_{\{r, s\} \subseteq\{1,2, \ldots, n\}}\left|\omega_{T}\left(v_{r}, v_{s}\right)\right|$,

(b) $|E|_{I}=\sum_{\{r, s\} \subseteq\{1,2, \ldots, n\}}\left|\omega_{I}\left(v_{r}, v_{s}\right)\right|$,

(c) $|E|_{F}=\sum_{\{r, s\} \subseteq\{1,2, \ldots, n\}}\left|\omega_{F}\left(v_{r}, v_{s}\right)\right|$.

On the other hand, sometimes we are particularly concerned about the number of non-void edges in an ordinary CNG1. In other words, we just want to know how many edges $\left\{v_{i}, v_{j}\right\}$ with:

$$
\omega\left(v_{i}, v_{j}\right) \neq(0,0,0) .
$$

Instead of a mere visual interpretation, we must however form a precise definition as follows:

Definition 15. Let $\xi=\langle V, \rho, \omega\rangle$ be an ordinary CNG1. Let $V=\left\{v_{1}, v_{2}, \ldots, v_{n}\right\}$ to be the vertex set of $\xi$. Then, the number of non-void edges of $\xi$, denoted as $M_{\xi}$, is defined to be the cardinality of the set:

$$
\left\{\left\{v_{i}, v_{j}\right\} \subseteq V \mid \omega\left(v_{i}, v_{j}\right) \neq(0,0,0)\right\} .
$$

Definition 16. Let $\xi=\langle V, \rho, \omega\rangle$ be an ordinary CNG1. Let $V=\left\{v_{1}, v_{2}, \ldots, v_{n}\right\}$ to be the vertex set of $\xi$. Then, the number of vertices of $\xi$, denoted as $N_{\tilde{\xi}}$, is defined to be the cardinality of the set $V$ itself. 
Remark 12. In this paper, we often deal with both $M_{\xi}$ and $N_{\xi}$ at the same time. Thus, we will not denote $N_{\xi}$ as $|V|$.

Remark 13. By Definition $7, V$ is non-void, so $N_{\xi} \geq 1$ follows.

Lemma 1. Let $\xi=\langle V, \rho, \omega\rangle$ be an ordinary CNG1. Let $V=\left\{v_{1}, v_{2}, \ldots, v_{n}\right\}$ be the vertex set of $\xi$. Then, for each $i$ :

(a) $D_{T}\left(v_{i}\right)=\sum_{r=1}^{n} \omega_{T}\left(v_{r}, v_{i}\right)+\omega_{T}\left(v_{i}, v_{i}\right)$,
(b) $D_{I}\left(v_{i}\right)=\sum_{r=1}^{n} \omega_{I}\left(v_{r}, v_{i}\right)+\omega_{I}\left(v_{i}, v_{i}\right)$,
(c) $D_{F}\left(v_{i}\right)=\sum_{r=1}^{n} \omega_{F}\left(v_{r}, v_{i}\right)+\omega_{F}\left(v_{i}, v_{i}\right)$.

Proof . Since $\xi$ is ordinary, $\omega\left(v_{r}, v_{i}\right)=\omega\left(v_{i}, v_{r}\right)$ for all $i$ and $r$. The lemma thus follows.

Lemma 2. Let $\xi=\langle V, \rho, \omega\rangle$ to be an ordinary CNG1. If $\xi$ is simple. then, for each $i$ :

(a) $D_{T}\left(v_{i}\right)=\sum_{r \in\{1,2, \ldots, n\}-\{i\}} \omega_{T}\left(v_{i}, v_{r}\right)$

(b) $D_{I}\left(v_{i}\right)=\sum_{r \in\{1,2, \ldots, n\}-\{i\}} \omega_{I}\left(v_{i}, v_{r}\right)$,

(c) $D_{F}\left(v_{i}\right)=\sum_{r \in\{1,2, \ldots, n\}-\{i\}} \omega_{F}\left(v_{i}, v_{r}\right)$.

Proof . Since $\xi$ is simple, $\omega\left(v_{i}, v_{i}\right)=(0,0,0)$ for all $i$. The lemma thus follows.

Lemma 3. Let $\xi=\langle V, \rho, \omega\rangle$ be an ordinary CNG1. Let $V=\left\{v_{1}, v_{2}, \ldots, v_{n}\right\}$ be the vertex set of $\xi$. Then, for each $i$ :

(a) $|D|_{T}\left(v_{i}\right)=\sum_{r=1}^{n}\left|\omega_{T}\left(v_{r}, v_{i}\right)\right|+\left|\omega_{T}\left(v_{i}, v_{i}\right)\right|$,

(b) $|D|_{I}\left(v_{i}\right)=\sum_{r=1}^{n}\left|\omega_{I}\left(v_{r}, v_{i}\right)\right|+\left|\omega_{I}\left(v_{i}, v_{i}\right)\right|$,

(c) $|D|_{F}\left(v_{i}\right)=\sum_{r=1}^{n}\left|\omega_{F}\left(v_{r}, v_{i}\right)\right|+\left|\omega_{F}\left(v_{i}, v_{i}\right)\right|$.

Proof. The arguments are similar to Lemma 1.

Lemma 4. Let $\xi=\langle V, \rho, \omega\rangle$ be an ordinary CNG1. If $\xi$ is simple. then, for each $i$ :

(a) $|D|_{T}\left(v_{i}\right)=\sum_{r \in\{1,2, \ldots, n\}-\{i\}}\left|\omega_{T}\left(v_{i}, v_{r}\right)\right|$,

(b) $|D|_{I}\left(v_{i}\right)=\sum_{r \in\{1,2, \ldots, n\}-\{i\}}\left|\omega_{I}\left(v_{i}, v_{r}\right)\right|$,

(c) $|D|_{F}\left(v_{i}\right)=\sum_{r \in\{1,2, \ldots, n\}-\{i\}}\left|\omega_{F}\left(v_{i}, v_{r}\right)\right|$.

Proof . The arguments are similar to Lemma 2.

Lemma 5. Let $\xi=\langle V, \rho, \omega\rangle$ be an ordinary CNG1. Then $\sum_{r=1}^{n}|D|\left(v_{r}\right)=(0,0,0)$ if and only if $|D|\left(v_{i}\right)=(0,0,0)$ for all $i$. 
Proof . Without loss of generality, since $|D|_{T}\left(v_{i}\right)=\sum_{r=1}^{n}\left|\omega_{T}\left(v_{i}, v_{r}\right)\right|+\left|\omega_{T}\left(v_{i}, v_{i}\right)\right|$ by Definition 13, it is always a non-negative real number. Thus, in order that $\sum_{r=1}^{n}|D|_{T}\left(v_{r}\right)=0$, there can be only one possibility: all $|D|_{T}\left(v_{i}\right)$ must be zero.

Remark 14. A similar statement does not hold for the resultant degree.

We now proceed with two of our theorems which both serve as generalizations of the well-known theorem in classical literature:

"For an ordinary graph, the sum of the degree of all its vertices is always twice the number of its edges".

Theorem 1. Let $\xi=\langle V, \rho, \omega\rangle$ be an ordinary CNG1. Then $\sum_{r=1}^{n} D\left(v_{r}\right)=2 E_{\tilde{\xi}}$.

Proof . As $D\left(v_{i}\right)=\left(D_{T}\left(v_{i}\right), D_{I}\left(v_{i}\right), D_{F}\left(v_{i}\right)\right)$ for all $i$, and $E_{\xi}=\left(E_{T}, E_{I}, E_{F}\right)$. Without loss of generality, it suffices to prove that $2 E_{T}=\sum_{r=1}^{n} D_{T}\left(v_{r}\right)$ :

$$
E_{T}=\sum_{\{r, s\} \subseteq\{1,2, \ldots, n\}} \omega_{T}\left(v_{r}, v_{s}\right)=\sum_{\{r, s\} \subseteq\{1,2, \ldots, n\}} \omega_{T}\left(v_{r}, v_{s}\right)+\sum_{r=1}^{n} \omega_{T}\left(v_{r}, v_{r}\right) .
$$

Since $\{r, s\}=\{s, r\}$ for all $s$ and $r$, it follows that:

$$
\begin{aligned}
2 E_{T}= & 2 \sum_{\substack{\{r, s\} \subseteq\{1,2, \ldots, n\} \\
r \neq s}} \omega_{T}\left(v_{r}, v_{s}\right)+2 \sum_{r=1}^{n} \omega_{T}\left(v_{r}, v_{r}\right) \\
= & \sum_{\substack{r \in\{1,2, \ldots, n\} \\
s \in\{1,2, \ldots, n\} \\
r \neq s}} \omega_{T}\left(v_{r}, v_{s}\right)+2 \sum_{r=1}^{n} \omega_{T}\left(v_{r}, v_{r}\right) \\
= & \sum_{\substack{r \in\{1,2, \ldots, n\} \\
s \in\{1,2, \ldots, n\}}} \omega_{T}\left(v_{r}, v_{s}\right)+\sum_{r=1}^{n} \omega_{T}\left(v_{r}, v_{r}\right) \\
= & \sum_{r=1}^{n} \sum_{s=1}^{n} \omega_{T}\left(v_{r}, v_{s}\right)+\sum_{r=1}^{n} \omega_{T}\left(v_{r}, v_{r}\right) \\
= & \sum_{r=1}^{n}\left(\sum_{s=1}^{n} \omega_{T}\left(v_{r}, v_{s}\right)+\omega_{T}\left(v_{r}, v_{r}\right)\right) \\
= & \sum_{r=1}^{n} D_{T}\left(v_{r}\right) .
\end{aligned}
$$

This completes the proof.

Theorem 2. Let $\xi=\langle V, \rho, \omega\rangle$ be an ordinary CNG1. Then $\sum_{r=1}^{n}|D|\left(v_{r}\right)=2|E|_{\xi}$.

Proof . The arguments are similar to Theorem 1 and can be easily proven by replacing all the terms $\omega_{T}\left(v_{i}, v_{j}\right)$ with $\left|\omega_{T}\left(v_{i}, v_{j}\right)\right|$.

Lemma 6. Let $\xi=\langle V, \rho, \omega\rangle$ be an ordinary CNG1, with $\sum_{r=1}^{n} D\left(v_{r}\right)=(0,0,0)$. If $M_{\xi}>0$, then $M_{\xi} \geq 2$.

Proof. By Theorem 1, $\sum_{r=1}^{n} D\left(v_{r}\right)=2 E_{\xi}$, so $E_{\xi}=(0,0,0)$ as well. 
If only one edge is non-void, then $\omega\left(v_{r_{0}}, v_{s_{0}}\right) \neq(0,0,0)$ only for one particular set $\left\{r_{0}, s_{0}\right\}$. This implies that:

$E_{T}=\sum_{\{r, s\} \subseteq\{1,2, \ldots, n\}} \omega_{T}\left(v_{r}, v_{s}\right)=\omega_{T}\left(v_{r_{0}}, v_{s_{0}}\right)$,

$E_{I}=\sum_{\{r, s\} \subseteq\{1,2, \ldots, n\}} \omega_{I}\left(v_{r}, v_{s}\right)=\omega_{I}\left(v_{r_{0}}, v_{s_{0}}\right)$,

$E_{F}=\sum_{\{r, s\} \subseteq\{1,2, \ldots, n\}} \omega_{F}\left(v_{r}, v_{s}\right)=\omega_{F}\left(v_{r_{0}}, v_{s_{0}}\right)$,

which contradicts the statement that $E_{\xi}=(0,0,0)$.

Since $M_{\xi} \geq 2$, one may have thought either $M_{\xi}$ or $N_{\xi}$ must be even. However, this is proven to be false, even by letting $\xi$ to be simple and by letting $D(v)=(0,0,0)$ for all $i$, as shown by the following counter-example (Figure 3):

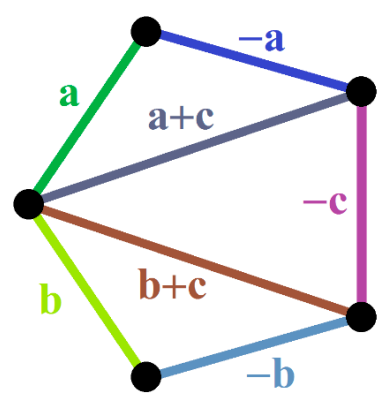

Figure 3. A counterexample, showing that $M_{\xi}$ or $N_{\xi}$ need not be even. $\mathbf{a}=\left(\frac{1}{5} \mathrm{e}^{\ell 2 \pi}, \frac{1}{5} \mathrm{e}^{\ell \frac{4}{3} \pi}, \frac{1}{5} \mathrm{e}^{\ell \frac{2}{3} \pi}\right)$, $\mathbf{b}=\left(\frac{1}{5} \mathrm{e}^{f \frac{4}{3} \pi}, \frac{1}{5} \mathrm{e}^{f \frac{2}{3} \pi}, \frac{1}{5} \mathrm{e}^{f 2 \pi}\right), \mathbf{c}=\left(\frac{1}{5} \mathrm{e}^{f \frac{2}{3} \pi}, \frac{1}{5} \mathrm{e}^{f 2 \pi}, \frac{1}{5} \mathrm{e}^{f \frac{4}{3} \pi}\right)$.

for which $M_{\xi}=7, N_{\xi}=5$, and with all vertices being end-points of some edges. Moreover, such a result is not related to the value of $\rho(v)$ for any of the vertex $v$.

This motivates to consider what is the least possible values of $M_{\xi}$ and $N_{\xi}$, for the special case of an ordinary $\xi$ being simple, with $D(v)=(0,0,0)$ and $\rho(v)=(1,0,0)$ for all of its vertices $v$.

\section{The Shortest CNG1 of Certain Conditions}

We now proceed with the following definitions.

Definition 17. Let $\xi=\langle V, \rho, \omega\rangle$ be an ordinary CNG1. $\xi$ is said to be net if all of the following are satisfied:

(a) $\xi$ is simple.

(b) $\xi$ is connected.

(c) for all $v \in V, D(v)=(0,0,0)$ and $\rho(v)=(1,0,0)$.

Furthermore, $\xi$ is said to be trivial if the entire $\xi$ consist of one single vertex $v$ with $\rho(v)=(1,0,0)$. On the other hand, $\xi$ is said to be gross if it is not net.

Lemma 7. Let $\xi=\langle V, \rho, \omega\rangle$ be a non-trivial net CNG1. Then each vertex must have least two non-void edges adjacent to it.

Proof . Let $v \in V$. Since $N_{\xi} \geq 2$ and $\xi$ is connected, there must exist a non-void edge $\{v, u\}$ for some $u \in V-\{v\}$.

If $\{v, u\}$ is the only non-void edge adjacent to $v$, then $D(v)=\omega(v, u) \neq(0,0,0)$. This a contradiction. 
Theorem 3. Let $\xi=\langle V, \rho, \omega\rangle$ be a non-trivial net CNG1. Then $M_{\xi} \geq 4$. Moreover, two of those non-void edges must be $\{a, b\}$ and $\{a, c\}$, for some mutually distinct vertices $a, b, c$.

Proof. Since $N_{\xi} \geq 2$ and $\xi$ is connected, non-void edge(s) must exist, so $M_{\xi}>0$. Furthermore, $\mathrm{D}(\mathrm{v})=(0,0,0)$ for all $v \in V$ would imply $\sum_{v \in V} D(v)=(0,0,0) . M_{\xi} \geq 2$ now follows by Lemma 6 .

Let $a$ be an end-point of some of those non-void edges. From Lemma 7, we conclude that at least two non-void edges must be adjacent to $a$.

Since $\xi$ is simple, it now follows that those 2 non-void edges must be $\{a, b\}$ and $\{a, c\}$, with $a, b, c$ being 3 mutually distinct vertices of $\xi$.

If $M_{\xi}=2$ :

$\{a, b\}$ and $\{a, c\}$ are therefore the only two non-void edges. By Lemma 7, both $\{a, b\}$ and $\{a, c\}$ must be adjacent to $b$. This is a contradiction.

If $M_{\xi}=3$ :

There can only be one more non-void edges besides $\{a, b\}$ and $\{a, c\}$.

By Lemma 7: $b$ must be an end-point of another non-void edge besides $\{a, b\}$; and $c$ must also be an end-point of another non-void edge besides $\{a, c\}$.

We now deduce that the third non-void edge must therefore be adjacent to both $b$ and $c$. This yields Figure 4:

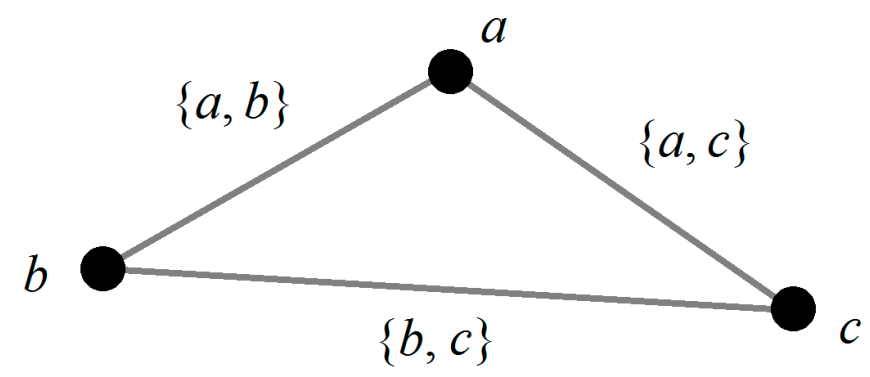

Figure 4. The triangle formed when $\{a, b\},\{a, c\}$ and $\{b, c\}$ are all non-void. Mentioned in Theorem 3,6 .

Since $\{a, b\}$ and $\{c, a\}$ are non void, $\omega(a, b)=\mathbf{k}=-\omega(c, a)$ for some $\mathbf{k} \neq(0,0,0)$.

Since $\{b, c\}$ is adjacent to both $b$ and $c, \omega(b, c)=\mathbf{k}=-\mathbf{k}$. This is again a contradiction. $M_{\xi} \geq 4$ now follows.

Theorem 4. Let $\xi=\langle V, \rho, \omega\rangle$ be a non-trivial net CNG1. Then $M_{\xi} \geq 4$ and $N_{\xi} \geq 4$.

Proof. By Theorem 3, $M_{\xi} \geq 4$, and two of those non-void edges must be $\{a, b\}$ and $\{a, c\}$, for some mutually distinct vertices $a, b, c$.

Suppose $N_{\xi}<4$. Since $\xi$ is simple, the maximum possible number of edges (whether it is void or not) is $3+\frac{3}{2}(3-3)=3<4$, which is a contradiction. $N_{\xi} \geq 4$ now follows.

Theorem 5. The smallest non-trivial net CNG1 must be of the structure in Figure 5:

Proof. Let $\xi=\langle V, \rho, \omega\rangle$ be a non-trivial net CNG1. By Theorem $4, M_{\xi} \geq 4$ and $N_{\xi} \geq 4$. By Theorem 3, two of those non-void edges must be $\{a, b\}$ and $\{a, c\}$, with $a, b, c$ being three mutually distinct vertices of $\xi$.

Consider the scenario where $M_{\xi}=4$ and $N_{\xi}=4$ (i.e., the least possible number).

If the edge $\{b, c\}$ is non-void, then we would have formed Figure 4 , as mentioned in the proof of Theorem 3 
That leaves us with only one vertex $d$ and only one extra non-void edge being adjacent to $d$. This is a contradiction.

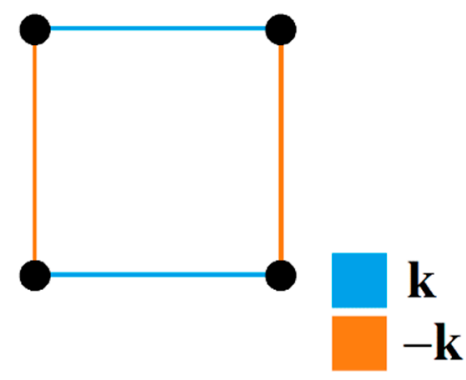

Figure 5. The smallest non-trivial net CNG1. Mentioned in Theorem 5 and Example $4 . \mathbf{k} \neq(0,0,0)$.

There is now only one choice left: both the edges $\{d, b\}$ and $\{d, c\}$ must be non-void. This gives rise to the following structure in Figure 6:

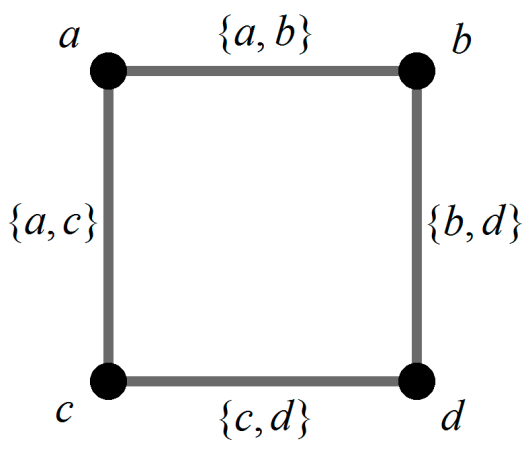

Figure 6. The only choices left because eace vertex must have at least 2 adjacent non void edges. Mentioned in Theorem 5, 6.

Without loss of generality, let $\omega(a, b)=\mathbf{k}$. Then both $\omega(b, d)=-\mathbf{k}$ and $\omega(a, c)=-\mathbf{k}$ must follow, leaving us with $\omega(c, d)=\mathbf{k}$ as the only valid option.

We are therefore left with the only way of assigning $\omega$ as shown by the theorem.

Lemma 8. Let $\xi=\langle V, \rho, \omega\rangle$ be a non-trivial net CNG1. Then $M_{\xi} \geq N_{\xi}$.

Proof . Every single non-void edge is connected to two vertices. Thus, if we count the total number of adjacent non-void edges for each vertex, and then summing the results for all the vertices together, the result will be $2 M_{\tilde{\zeta}}$ (note: this paragraph is analogous to classical graph theory).

By Lemma 7, each vertex must have at least two non-void edges connect to it. We now have $2 M_{\tilde{\zeta}} \geq 2 N_{\tilde{\xi}}$, so $M_{\tilde{\zeta}} \geq N_{\xi}$ follows.

Theorem 6. Let $\xi=\langle V, \rho, \omega\rangle$ be a non-trivial net CNG1 with both $M_{\xi}$ and $N_{\xi}$ being odd numbers. Then $M_{\xi} \geq 7$ and $N_{\xi} \geq 5$.

Proof. Let $\xi=V, \rho, \omega$ be a non-trivial net CNG1. By Theorem $4, M_{\xi} \geq 4$ and $N_{\xi} \geq 4$. By Theorem 3 , two of those non-void edges must be $\{a, b\}$ and $\{a, c\}$, for some $a, b$ and $c$ being three mutually distinct vertices of $\xi$.

Since both $M_{\xi}$ and $N_{\xi}$ are odd, it follows that $M_{\xi} \geq 5$ and $N_{\xi} \geq 5$. So in addition to $a, b, c$, there exist another 2 vertices $d, e$. 
Consider the scenario where $M_{\xi}=5$ and $N_{\xi}=5$ (i.e., the least possible number).

Case 1. Suppose the edge $\{b, c\}$ is non-void. Then we would have formed Figure 4, as mentioned in the proof of Theorem 3.

That leaves us with two vertices $d$ and $e$, and two extra non-void edge, which both of them must be adjacent to $d$. Even if $\{d, e\}$ is non-void, the other non-void edge adjacent to $d$ cannot possibly be $\{d, e\}$ itself. Therefore, we have, at most, one non-void edge being adjacent to $e$. This is a contradiction.

Case 2. Without loss of generality, suppose the edges $\{b, d\}$ and $\{c, d\}$ are non-void. Then we would have formed Figure 6, as mentioned in the proof of Theorem 5.

That leaves us with only one vertex $e$ and only one extra edge being adjacent to $e$, which is, again, a contradiction.

Case 3. Without loss of generality, suppose the edges $\{b, d\}$ and $\{c, e\}$ are non-void. Then, besides $\{b, d\}$, another edge must be adjacent to $d$. Likewise, besides $\{c, e\}$, another edge must be adjacent to $e$. Since we are left with one edge, it must, therefore, be $\{d, e\}$. This gives rise to the following structure in Figure 7:

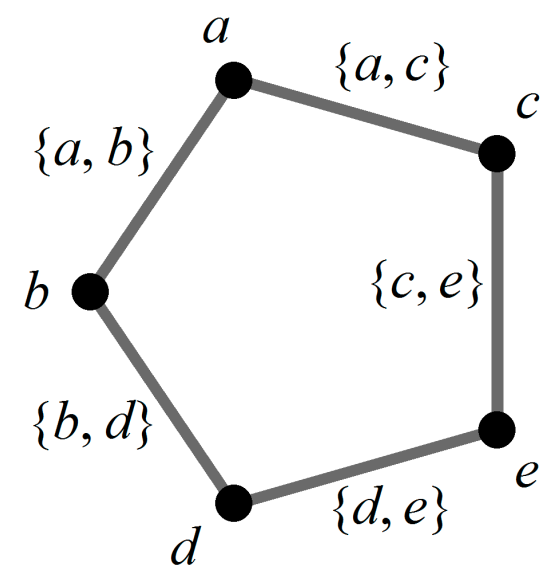

Figure 7. The only choice left for the case of 5 non-void edges connecting to 5 vertices. Mentioned in Theorem 6.

Without loss of generality, let $\omega(a, b)=\mathbf{k}$. Then both $\omega(b, d)=-\mathbf{k}$ and $\omega(a, c)=-\mathbf{k}$ must follow, leaving us with both $\omega(c, e)=\mathbf{k}$ and $\omega(d, e)=\mathbf{k}$.

We have, thus, arrived at $D(e)=2 \mathbf{k} \neq(0,0,0)$, again a contradiction.

Hence, it is either $M_{\tilde{\zeta}}>5$ or $N_{\xi}>5$.

Since both $M_{\xi}$ and $N_{\xi}$ are odd, either one of the following must hold:

(a) $\quad M_{\xi} \geq 7$ and $N_{\xi} \geq 7$.

(b) $M_{\xi}=7$ and $N_{\xi}=5$.

(c) $\quad M_{\xi}=5$ and $N_{\xi}=7$.

Furthermore, by Lemma $8, M_{\xi} \geq N_{\xi}$. Hence (c) will not occur, which implies that $M_{\xi} \geq 7$ and $N_{\xi} \geq 5$. This completes the proof.

Theorem 7. The smallest non-trivial net CNG1 $\xi$, with both $M_{\xi}$ and $N_{\xi}$ being odd numbers, must be of the structure as shown in Figure 8:

Proof . Let $\xi=\langle V, \rho, \omega\rangle$ be a non-trivial net CNG1 with both $M_{\xi}$ and $N_{\xi}$ being odd numbers. Then $M_{\xi} \geq 7$ and $N_{\xi} \geq 5$.

Consider the scenario where $M_{\tilde{\xi}}=7$ and $N_{\tilde{\xi}}=5$ (i.e., the least possible number). 
Since $\xi$ is an ordinary CNG1, each vertex must have 5 edges adjacent to it (whether void or not). Since $\xi$ is simple, one of the five edges for each vertex, which is a loop, must be void. As a result, we now conclude that each vertex must have at most 4 non-void edges adjacent to it.

On the other hand, by Lemma 7, each vertex must have at least two non-void edges adjacent to it.

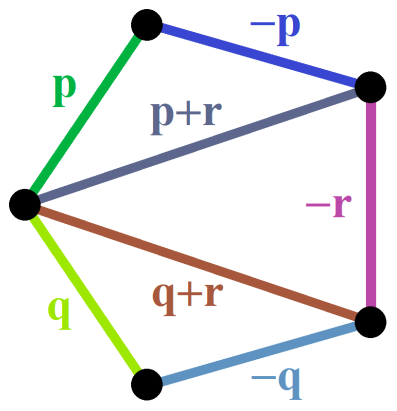

Figure 8. The smallest non-trivial net $\mathrm{CNG1}$, with both $\mathrm{M}_{\xi}$ and $\mathrm{N}_{\xi}$ being odd numbers. . Mentioned in Theorem 7 and Example 5. $\mathbf{p}+\mathbf{q}+\mathbf{r}=(0,0,0) ;|\mathbf{p}+\mathbf{r}|,|\mathbf{q}+\mathbf{r}| \leq 1$.

Since every single non-void edge is adjacent to two vertices. Thus, if we count the total number of adjacent non-void edges for each vertex, and then summing the results for all the vertices together, the result will be $7 \times 2=14$ (note: this paragraph is analogous to classical graph theory).

Hence, the set representing the number of non-void edges adjacent to each of the five vertices, must be one of the following:

(a) $\{2,3,3,3,3\}$ (most "widely spread" possibility)

(b) $\{2,2,3,3,4\}$

(c) $\{2,2,2,4,4\}$ (most "concentrated" possibility)

We now consider each the three cases:

Case 1. $\{2,3,3,3,3\}$

Without loss of generality:

Let $a$ be that one vertex which is an end-point to only 2 non-void edges $\{a, b\}$ and $\{a, c\}$. (i.e., $\{a, d\},\{a, e\}$ are void)(Figure 9).

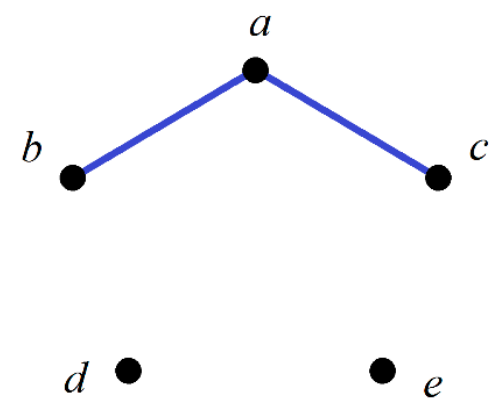

Figure 9. The 2 non-void edges $\{a, b\}$ and $\{a, c\}$, for all the 3 cases of Theorem 7 .

Then, each one among $b, c, d, e$ must be an end-point of three non-void edges.

Besides $\{d, a\}$ and $\{d, d\}$, which are both void, there are three more edges adjacent to $d:\{d, b\}$, $\{d, c\},\{d, e\}$. 
Since $d$ is an end-point of exactly three non-void edges, we conclude that:

$$
\{d, b\},\{d, c\},\{d, e\} \text { areallnon - void }
$$

Similarly, besides $\{e, a\}$ and $\{e, e\}$, which are both void, there are three more edges adjacent to $e$ : $\{e, b\},\{e, c\},\{e, d\}$.

Since $e$ is also an end-point of exactly three non-void edges, we conclude that:

$$
\{e, b\},\{e, c\},\{e, d\} \text { areallnon - void }
$$

From (10) and (11), we conclude that:

$$
\{d, b\},\{d, c\},\{e, b\},\{e, c\},\{d, e\}=\{e, d\} \text { areallnon }- \text { void }
$$

From (9) and (12), we have obtained all the seven non-void edges:

$$
\{d, b\},\{d, c\},\{e, b\},\{e, c\},\{d, e\},\{a, b\},\{a, c\} \text {. }
$$

Hence, $\{b, c\},\{a, d\},\{a, e\}$ must be all void. We, thus, obtain the following structure (Figure 10):

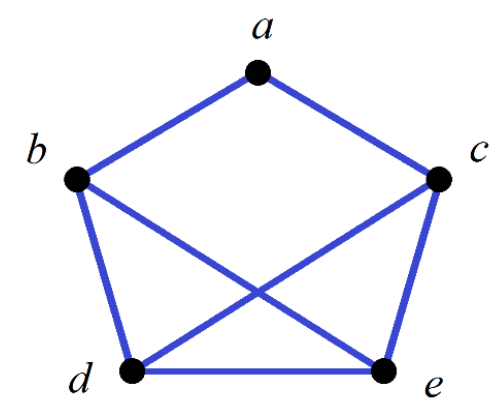

Figure 10. The only possible way of connection for $\{2,3,3,3,3\}$.

Let $\omega(a, b)=\mathbf{p}, \omega(b, d)=\mathbf{q}, \omega(b, e)=\mathbf{r}, \omega(c, d)=\mathbf{s}, \omega(c, e)=\mathbf{t}, \omega(d, e)=\mathbf{u}$.

Since $\{a, b\}$ and $\{a, c\}$ are the only two non-void edges adjacent to $a$, we now have $\omega(a, c)=-\mathbf{p}$ (Figure 11).

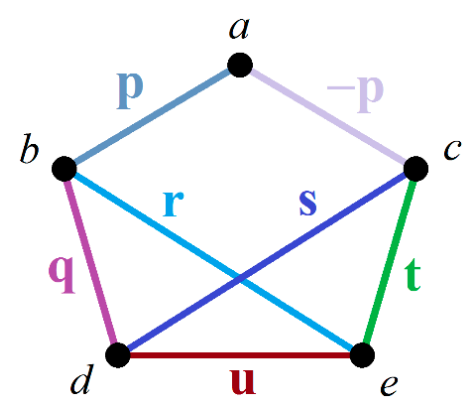

Figure 11. The labeling of the non-void edges for $\{2,3,3,3,3\}$.

We how have: $\mathbf{r}+\mathbf{q}+\mathbf{p}=\mathbf{s}+\mathbf{t}-\mathbf{p}=\mathbf{s}+\mathbf{q}+\mathbf{u}=\mathbf{r}+\mathbf{t}+\mathbf{u}=(0,0,0)$.

This further implies that: $\mathbf{r}+\mathbf{q}+\mathbf{p}+\mathbf{s}+\mathbf{t}-\mathbf{p}=\mathbf{s}+\mathbf{q}+\mathbf{u}+\mathbf{r}+\mathbf{t}+\mathbf{u}=(0,0,0)$.

Therefore, $\mathbf{q}+\mathbf{r}+\mathbf{s}+\mathbf{t}=\mathbf{q}+\mathbf{r}+\mathbf{s}+\mathbf{t}+2 \mathbf{u}$, which implies that $\mathbf{u}=(0,0,0)$. This is a contradiction.

Case 2. $\{2,2,3,3,4\}$ 
Without loss of generality:

Let $a$ be that one vertex which is an end-point to only two non-void edges $\{a, b\}$ and $\{a, c\}$. (i. e., $\{a, d\},\{a, e\}$ are void)

as shown in Figure 9.

Since $\{a, d\},\{a, e\}$ are void, both $d$ and $e$ cannot be that vertex which is an end-point to four non-void edges.

By symmetry, fix $b$ to be that vertex which is an end-point to four non-void edges. Then:

$$
\{b, a\},\{b, c\},\{b, d\},\{b, e\} \text { areallnon - void. }
$$

From (13) and (14), we have now arrived at the following structure (Figure 12):

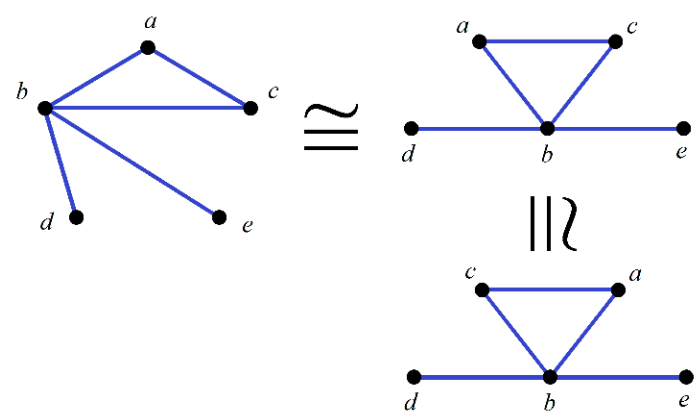

Figure 12. The first 5 non-void edges for $\{2,2,3,3,4\}$.

Suppose $\{d, e\}$ is void. Then exactly one out of $\{d, c\}$ and $\{d, a\}$ must be non-void. Similarly, exactly one out of $\{e, c\}$ and $\{e, a\}$ must be non-void. By symmetry and the rules of graph isomorphism, fix $\{d, a\}$ to be non-void, then $a$ would have been an end-point of three non-void edges: $\{d, a\},\{b, a\}$, $\{c, a\}$. So $\{e, a\}$ must be void and, therefore, $\{e, c\}$ is non-void. We, thus, obtain the following structure (Figure 13):

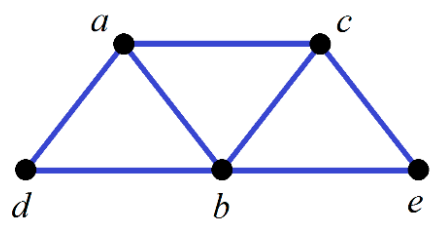

Figure 13. The only possible way of connection for $\{2,2,3,3,4\}$, if $\{d, e\}$ is void.

Suppose $\{d, e\}$ is non-void. Then we now arrived at the following structure (Figure 14):
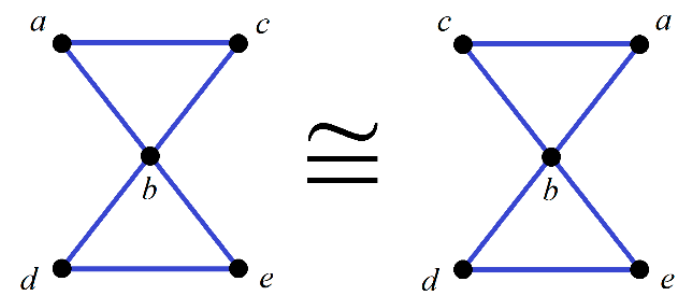

Figure 14. The first 6 non-void edges for $\{2,2,3,3,4\}$, for the case of non-void $\{d, e\}$. 
By symmetry, fix $a$ to be a vertex which is an end-point of three non-void edges. Then exactly one edge out of $\{a, d\}$ and $\{a, e\}$ must be non-void. By the rules of graph isomorphism, we can fix $\{a, d\}$ to be non-void. Again we obtain the following structure (Figure 15):

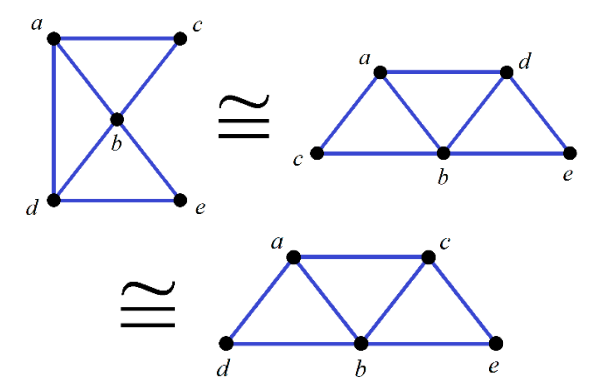

Figure 15. The only possible way of connection for $\{2,2,3,3,4\}$, if $\{d, e\}$ is non-void.

Let $\omega(a, b)=\mathbf{g}, \omega(c, b)=\mathbf{h}, \omega(a, c)=\mathbf{k}, \omega(b, d)=\mathbf{p}, \omega(b, e)=\mathbf{q}$.

Since $\{a, d\}$ and $\{b, d\}$ are the only two non-void edges adjacent to $d$, we now have $\omega(a, d)=-\mathbf{p}$.

Likewise, since $\{c, e\}$ and $\{b, e\}$ are the only two non-void edges adjacent to $e$, we now have $\omega(c, e)=-\mathbf{q}$ (Figure 16).

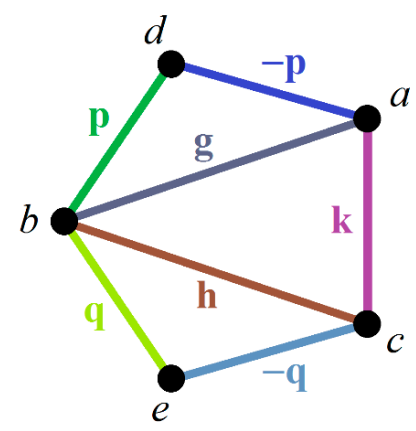

Figure 16. The labeling of the non-void edges for $\{2,2,3,3,4\}$.

We how have: $\mathbf{p}+\mathbf{q}+\mathbf{g}+\mathbf{h}=\mathbf{g}+\mathbf{k}-\mathbf{p}=\mathbf{h}+\mathbf{k}-\mathbf{q}=(0,0,0)$.

Therefore, $\mathbf{g}=\mathbf{p}-\mathbf{k}, \mathbf{h}=\mathbf{q}-\mathbf{k}$. As a result: $\mathbf{p}+\mathbf{q}+\mathbf{p}-\mathbf{k}+\mathbf{q}-\mathbf{k}=2 \mathbf{p}+2 \mathbf{q}-2 \mathbf{k}=(0,0,0)$, which implies $\mathbf{p}+\mathbf{q}-\mathbf{k}=(0,0,0)$.

Denote $-\mathbf{k}=\mathbf{r}$. Then $\mathbf{g}=\mathbf{p}+\mathbf{r}, \mathbf{h}=\mathbf{q}+\mathbf{r}$, and $\mathbf{p}+\mathbf{q}+\mathbf{r}=(0,0,0)$ follows. We have, thus, formed the structure as mentioned in this theorem.

Case 3. $\{2,2,2,4,4\}$

Without loss of generality:

Let $a$ be one of that two vertices which is an end-point to four non-void edges. Then:

$$
\{a, b\},\{a, c\},\{a, d\},\{a, e\} \text { arenon - void. }
$$

Let $b$ be the other one vertices which is also an end-point to four non-void edges. Then:

$$
\{b, a\},\{b, c\},\{b, d\},\{b, e\} \text { arenon - void. }
$$

From (15) and (16), we have obtained the seven non-void edges:

$$
\{a, c\},\{a, d\},\{a, e\},\{b, c\},\{b, d\},\{b, e\},\{a, b\} .
$$

Hence, $\{c, d\},\{c, e\},\{d, e\}$ are all void. We, thus, obtain the following structure (Figure 17): 


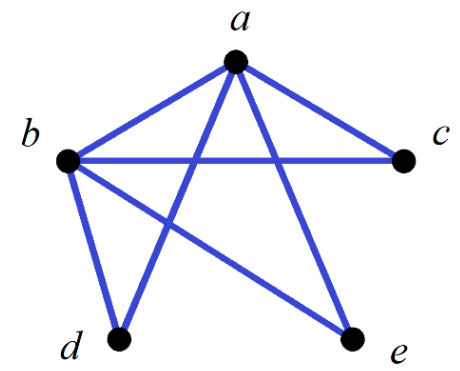

Figure 17. The only possible way of connection for $\{2,2,2,4,4\}$.

Let $\omega(b, d)=\mathbf{p}, \omega(b, e)=\mathbf{q}, \omega(b, c)=\mathbf{r}, \omega(b, a)=\mathbf{s}$,

Since $\{a, d\}$ and $\{b, d\}$ are the only two non-void edges adjacent to $d$, we now have $=-\mathbf{p}$.

Since $\{a, e\}$ and $\{b, e\}$ are the only two non-void edges adjacent to $e$, we now have $\omega(a, e)=-\mathbf{q}$.

Since $\{a, c\}$ and $\{b, c\}$ are the only two non-void edges adjacent to $c$, we now have $\omega(a, c)=-\mathbf{r}$ (Figure 18).

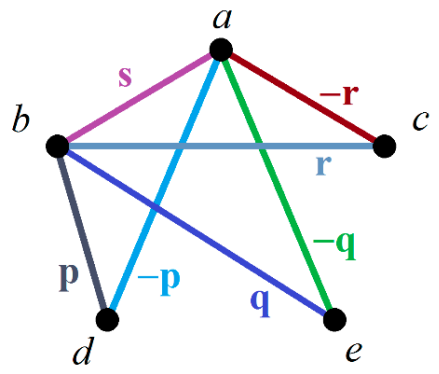

Figure 18. The labeling of the non-void edges for $\{2,2,2,4,4\}$.

We how have: $\mathbf{s}+\mathbf{p}+\mathbf{q}+\mathbf{r}=\mathbf{s}-\mathbf{p}-\mathbf{q}-\mathbf{r}=(0,0,0)$.

This further implies that: $\mathbf{s}+\mathbf{p}+\mathbf{q}+\mathbf{r}+\mathbf{s}-\mathbf{p}-\mathbf{q}-\mathbf{r}=(0,0,0)$.

We now have $2 \mathbf{s}=(0,0,0)$, which implies that $\mathbf{s}=(0,0,0)$. This is a contradiction.

Our proof is now complete.

Note that both 5 and 7 are not divisible even by 3, the next prime number after 2 . This yields the following corollary:

Corollary 1. The smallest non-trivial net CNG1 $\xi$, with both $M_{\xi}$ and $N_{\xi}$ not divisible by 2 or 3 , must also be of the structure as shown in Figure:

\section{Symmetric Properties of Ordinary Simple CNG1}

Definition 18. Let $V$ and $W$ be two non-void sets. Let $\xi=\langle V, \rho, \omega\rangle$ and $\zeta=\langle W, \zeta, \psi\rangle$ be two ordinary CNG1s. If $V=W, \rho=\varsigma$ and $\omega=\psi$, then $\xi$ and $\zeta$ are said to be equal, and shall be denoted by $\xi \equiv \zeta$.

Definition 19. Let $V$ and $W$ be a non-void set. Let $\xi=\langle V, \rho, \omega\rangle$ and $\zeta=\langle W, \varsigma, \psi\rangle$ be two ordinary CNG1s. If there exist a bijection $f: V \rightarrow W$ such that:

(a) $\rho(u)=\varsigma(\mathcal{f}(u))$ for all $u \in V$.

(b) $\omega(u, v)=\psi(f(u), f(v))$ for all $u, v \in V$.

Then: 
(i) Such $f$ is said to be an isomorphism from $\xi$ to $\zeta$, and we shall denote such case by $f[\xi] \equiv \zeta$.

(ii) $\xi$ and $\zeta$ are said to be isomorphic, and shall be denoted by $\xi \cong \zeta$.

Remark 15. As both $\xi$ and $\zeta$ are ordinary, $\omega(u, v)=\omega(v, u)$ and $\psi(f(u), f(v))=\psi(f(v), f(u))$ follow for all $u, v \in V$.

Example 1. Consider $\xi_{0}=V_{0}, \rho_{0}, \omega_{0}$ and $\zeta_{0}=W_{0}, \varsigma_{0}, \psi_{0}$ as follows:

$V_{0}=\left\{v_{1}, v_{2}, v_{3}, v_{4}, v_{5}, v_{6}\right\} . W_{0}=\left\{w_{1}, w_{2}, w_{3}, w_{4}, w_{5}, w_{6}\right\}$.

$\rho_{0}\left(v_{1}\right)=\mathbf{p}, \rho_{0}\left(v_{2}\right)=\mathbf{q}, \rho_{0}\left(v_{3}\right)=\mathbf{t}, \varsigma_{0}\left(w_{1}\right)=\mathbf{t}, \varsigma_{0}\left(w_{3}\right)=\mathbf{p}, \varsigma_{0}\left(w_{4}\right)=\mathbf{s}$,

$\rho_{0}\left(v_{4}\right)=\rho_{0}\left(v_{6}\right)=\mathbf{r}, \rho_{0}\left(v_{5}\right)=\mathbf{s} . \varsigma_{0}\left(w_{2}\right)=\varsigma_{0}\left(w_{4}\right)=\mathbf{r}, \varsigma_{0}\left(w_{6}\right)=\mathbf{q}$.

$\omega_{0}\left(v_{1}, v_{2}\right)=\omega_{0}\left(v_{2}, v_{1}\right)=\omega_{0}\left(v_{2}, v_{2}\right)=\mathbf{a}, \psi_{0}\left(w_{1}, w_{2}\right)=\psi_{0}\left(w_{2}, w_{1}\right)=\mathbf{d}$,

$\omega_{0}\left(v_{1}, v_{3}\right)=\omega_{0}\left(v_{3}, v_{1}\right)=\mathbf{c}, \psi_{0}\left(w_{1}, w_{3}\right)=\psi_{0}\left(w_{3}, w_{1}\right)=\mathbf{c}$,

$\omega_{0}\left(v_{2}, v_{3}\right)=\omega_{0}\left(v_{3}, v_{2}\right)=\mathbf{b}, \psi_{0}\left(w_{1}, w_{4}\right)=\psi_{0}\left(w_{4}, w_{1}\right)=\mathbf{e}$,

$\omega_{0}\left(v_{3}, v_{4}\right)=\omega_{0}\left(v_{4}, v_{3}\right)=\mathbf{d}, \psi_{0}\left(w_{4}, w_{5}\right)=\psi_{0}\left(w_{5}, w_{4}\right)=\mathbf{f}$,

$\omega_{0}\left(v_{3}, v_{5}\right)=\omega_{0}\left(v_{5}, v_{3}\right)=\mathbf{e}, \psi_{0}\left(w_{1}, w_{6}\right)=\psi_{0}\left(w_{6}, w_{1}\right)=\mathbf{b}$,

$\omega_{0}\left(v_{5}, v_{6}\right)=\omega_{0}\left(v_{6}, v_{5}\right)=\mathbf{f}, \psi_{0}\left(w_{3}, w_{6}\right)=\psi_{0}\left(w_{6}, w_{3}\right)=\psi_{0}\left(w_{6}, w_{6}\right)=\mathbf{a}$,

otherwise, $\omega_{0}(u, v)=(0,0,0)$. otherwise, $\psi_{0}(w, v)=(0,0,0)$.

Moreover, $|\{\mathbf{p}, \mathbf{q}, \mathbf{r}, \mathbf{s}, \mathbf{t}\}|=5$ and $|\{\mathbf{a}, \mathbf{b}, \mathbf{c}, \mathbf{d}, \mathbf{e}, \mathbf{f}\}|=6$ (Figure 19).

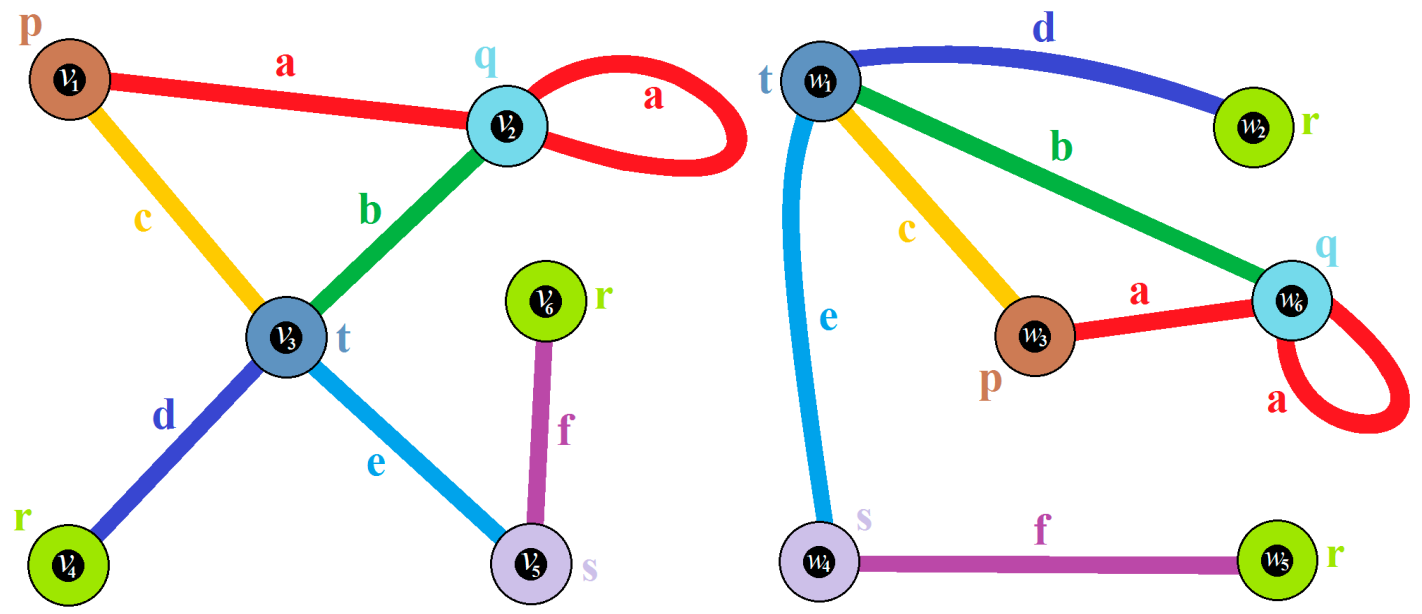

Figure 19. Two isomorphic CNG1's, as mentioned in Example 1.

Thus, we define the bijection $f_{0}: V \rightarrow W$ as:

$$
f_{0}\left(v_{1}\right)=v_{3}, f_{0}\left(v_{2}\right)=v_{6}, f_{0}\left(v_{3}\right)=v_{1}, f_{0}\left(v_{4}\right)=v_{2}, f_{0}\left(v_{5}\right)=v_{4}, f_{0}\left(v_{6}\right)=v_{5} .
$$

It now follows that $f_{0}$ is an isomorphism from $\xi_{0}$ to $\zeta_{0}$, so $\xi_{0} \cong \zeta_{0}$. Still, $\xi_{0} \zeta_{0}$ in accordance with Definition 18.

In all the following passages of this paper, let $\mathscr{I}: V \rightarrow V$ be the identity mapping from $V$ to itself.

Like classical graph theory, whenever $\zeta \equiv \xi, \mathscr{I}$ is an isomorphism from $\xi$ to $\xi$ itself in accordance with Definition 19. It is, therefore, motivational to investigate if there are other non-identity bijections from $V$ to itself, which is also an isomorphism from $\xi$ to $\xi$ itself. Additionally, recall that, in classical graph theory, an isomorphism from a graph to itself will be called an automorphism on that graph. Thus, we proceed with the following definition: 
Definition 20. Let $V$ be a non-void set. Let $\xi=\langle V, \rho, \omega\rangle$ be an ordinary CNG1's. Let $f: V \rightarrow V$ be a bijection such that:

(a) $\rho(u)=\rho(f(u))$ for all $u \in V$.

(b) $\omega(u, v)=\omega(f(u), f(v))$ for all $u, v \in V$.

Then $f$ is said to be an automorphism of $\xi$.

Remark 16. As $\xi$ is ordinary, $\omega(u, v)=\omega(v, u)$ follows for all $u, v \in V$.

Remark 17. Just because $\rho(u)=\rho(f(u))$ and $\omega(u, v)=\omega(f(u), f(v))$, does not mean that $u=f(u)$ or $v=f(v)$.

Remark 18. $\mathscr{I}$ is thus called the trivial automorphism of $\xi$.

Example 2. Consider $\xi_{1}=\left\langle V_{1}, \rho_{1}, \omega_{1}\right\rangle$ as shown in Figure 20:

$V_{1}=\{a, b, c, d\} \cdot \rho_{1}(a)=\rho_{1}(b)=\rho_{1}(d)=\mathbf{p}, \rho_{1}(c)=\mathbf{q}$.

$\omega_{1}(a, c)=\omega_{1}(c, a)=\mathbf{h}, \omega_{1}(b, c)=\omega_{1}(c, b)=\omega_{1}(d, c)=\omega_{1}(c, d)=\mathbf{g}$, otherwise, $\omega_{1}(u, v)=(0,0,0)$.

$|\{\mathbf{p}, \mathbf{q}\}|=|\{\mathbf{g}, \mathbf{h}\}|=2$.

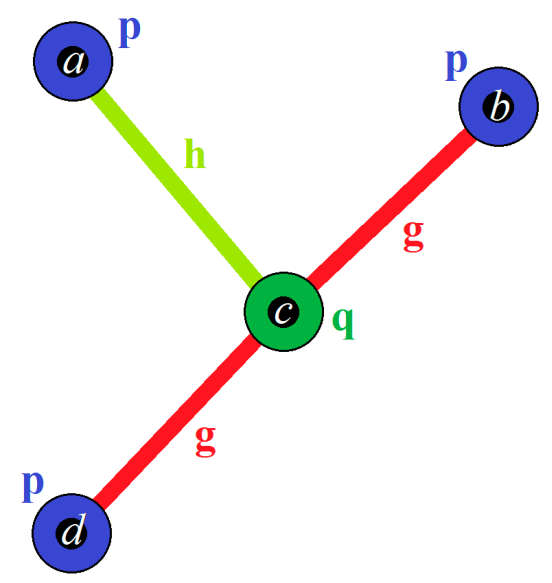

Figure 20. $\xi_{1}$ as mentioned in Example 2.

Let $f_{1}, b_{1}, h_{1}: V_{1} \rightarrow V_{1}$ be three bijections defined as follows:

(a) $f_{1}(c)=a, f_{1}(a)=d, f_{1}(d)=c, f_{1}(b)=b$.

(b) $b_{1}(b)=a, b_{1}(d)=d, b_{1}(c)=c, b_{1}(a)=b$.

(c) $h_{1}(b)=d, h_{1}(a)=a, h_{1}(c)=c, h_{1}(d)=b$.

Then:

(i) $f_{1}$ is an isomorphism from $V_{1}$ to the following ordinary CNG1 (Figure 21).

which is not equal to $\xi_{1}$ in accordance with Definition $18 . f_{1}$ is therefore not an automorphism of $\xi_{1}$.

(ii) $b_{1}$ is an isomorphism from $V_{1}$ to the following ordinary CNG1 (Figure 22).

which is also not equal to $\xi_{1}$ in accordance with Definition 18. Likewise $b_{1}$ is, therefore, not an automorphism of $\xi_{1}$.

(iii) $h_{1}$ is an isomorphism from $V_{1}$ to itself and, therefore, it is an automorphism of $\xi_{1}$. Note that, even if $h_{1}(b)=d$ and $h_{1}(d)=b$, as $\rho_{1}(b)=\rho_{1}(d)=\mathbf{p}$ and $\omega_{1}(b, c)=\omega_{1}(d, c)=\mathbf{g}$, so $h_{1}\left[\xi_{1}\right] \equiv \xi_{1}$ still holds. 


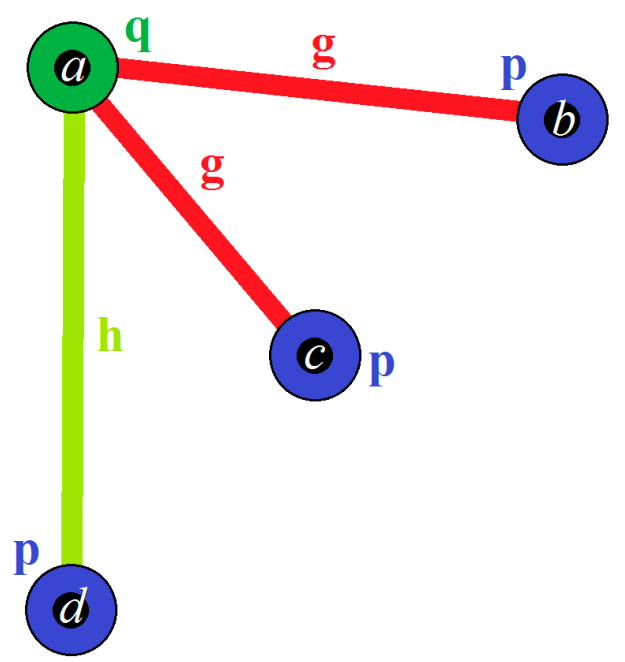

Figure 21. This is not an automorphism of $\xi_{1}$ as mentioned in Example 2.

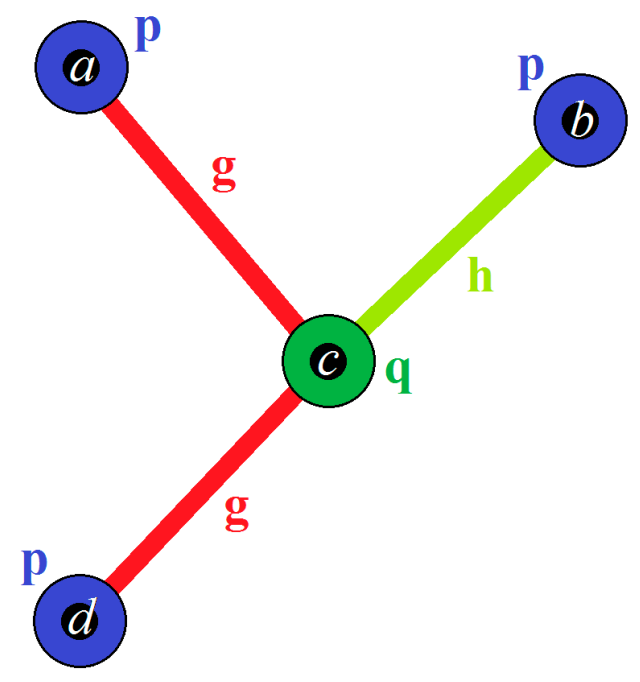

Figure 22. This is not an automorphism of $\xi_{1}$ as mentioned in Example 2.

Definition 21. Let $\xi=\langle V, \rho, \omega\rangle$ be an ordinary simple CNG1. $\xi$ is said to be total symmetric if, for all $\left\{u_{1}, v_{1}\right\},\left\{u_{2}, v_{2}\right\} \subseteq V$, with $\left|\left\{u_{1}, v_{1}\right\}\right|=\left|\left\{u_{2}, v_{2}\right\}\right|$, there exist an automorphism of $\xi$, $f$, such that $u_{2}=f\left(u_{1}\right), v_{2}=f\left(v_{1}\right)$.

Remark 19. In other words, $\left\{u_{1}, v_{1}\right\},\left\{u_{2}, v_{2}\right\}$ can either be two edges, or two vertices as when $u_{1}=v_{1}$ and $u_{2}=v_{2}$.

Example 3. With this definition, the following CNG1 (Figure 23) is, thus, totally-symmetric.

However, unlike symmetry of classical graphs, the concept of total symmetry takes all the edges into account, whether void or not. As a result, the following graph (Figure 24), though looks familiar to the classical literature, is not totally-symmetric.

As a result, the concept of total-symmetry in ordinary simple CNG1 proves even more stringent than the concept of symmetry in classical ordinary simple graphs. Additionally, recall that edges and vertices in CNG1 have three membership values instead of only 0 (disconnected, void) and 1 
(connected). To give more characterization of symmetry among ordinary simple CNG1, we now proceed with the following definitions.

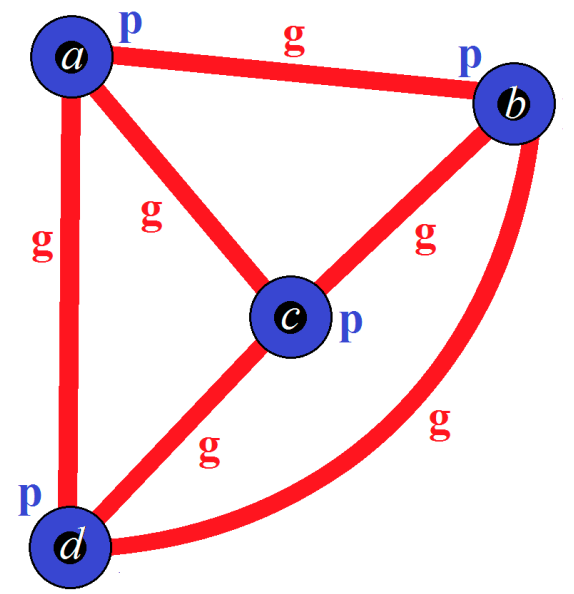

Figure 23. A totally-symmetric CNG1, as mentioned in Example 3.
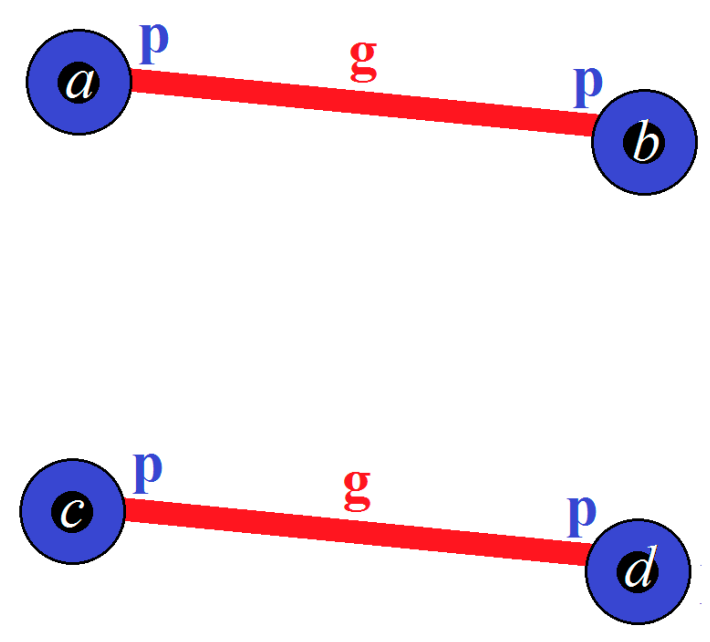

Figure 24. This graph is not totally-symmetric. Mentioned in Example 3.

Definition 22. Let $\xi=\langle V, \rho, \omega\rangle$ be an ordinary simple CNG1. $\xi$ is said to be strong edge-wise symmetric (abbr. SES) if: For all $\left\{u_{1}, v_{1}\right\},\left\{u_{2}, v_{2}\right\} \subseteq V$ with both $\omega\left(u_{1}, v_{1}\right)$ and $\omega\left(u_{2}, v_{2}\right)$ non-void, there exist an automorphism $f$ of $\xi$, such that $u_{2}=f\left(u_{1}\right), v_{2}=f\left(v_{1}\right)$.

Remark 20. As $\xi$ is simple, it follows that $\left|\left\{u_{1}, v_{1}\right\}\right|=\left|\left\{u_{2}, v_{2}\right\}\right|=2$.

Remark 21. An ordinary simple CNG1 with all edges being void is classified as strong edge-wise symmetric as well.

Definition 23. Let $\xi=\langle V, \rho, \omega\rangle$ be an ordinary simple CNG1. $\xi$ is said to be strong point-wise(or vertex-wise) symmetric (abbr. SPS) if: For all $u_{1}, u_{2} \in V$ with both $\rho\left(u_{1}\right)$ and $\rho\left(u_{2}\right)$ non-void, there exists an automorphism $f$ of $\xi$, such that $u_{2}=f\left(u_{1}\right)$.

Remark 22. An ordinary simple CNG1 with all vertices being void is classified as strong point-wise symmetric as well. 
Definition 24. Let $\xi=\langle V, \rho, \omega\rangle$ be an ordinary simple CNG1. $\xi$ is said to be strong symmetric (abbr. SS) if it is both strong edge-wise symmetric and strong point-wise symmetric.

Definition 25. Let $\xi=\langle V, \rho, \omega\rangle$ be an ordinary simple $C N G 1$. $\xi$ is said to be weak edge-wise symmetric (abbr. $w E S)$ if: For all $\left\{u_{1}, v_{1}\right\},\left\{u_{2}, v_{2}\right\} \subseteq V$ with $\omega\left(u_{1}, v_{1}\right)=\omega\left(u_{2}, v_{2}\right) \neq(0,0,0)$, there exists an automorphism $f$ of $\xi$, such that $u_{2}=f\left(u_{1}\right), v_{2}=f\left(v_{1}\right)$. Otherwise, $\xi$ is said to be edge-wise asymmetric (abbr. EA).

Remark 23. Again, as $\xi$ is simple, it follows that $\left|\left\{u_{1}, v_{1}\right\}\right|=\left|\left\{u_{2}, v_{2}\right\}\right|=2$.

Remark 24. An ordinary simple CNG1 with all non-void edges having different membership value is classified as weak edge-wise symmetric as well.

Definition 26. Let $\xi=V, \rho, \omega$ be an ordinary simple CNG1. $\xi$ is said to be weak point-wise (or vertex-wise) symmetric (abbr. wPS) if: For all $u_{1}, u_{2} \in V$ with $\rho\left(u_{1}\right)=\rho\left(u_{2}\right) \neq(0,0,0)$, there exists an automorphism $f$ of $\xi$, such that $u_{2}=f\left(u_{1}\right)$. Otherwise, $\xi$ is said to be point-wise asymmetric (abbr. PA).

Remark 25. An ordinary simple CNG1 with all non-void vertices having different membership value is classified as weak point-wise symmetric as well.

Definition 27. Let $\xi=\langle V, \rho, \omega\rangle$ be an ordinary simple CNG1. $\xi$ is said to be asymmetric if it is both edge-wise asymmetric and point-wise asymmetric.

Based on the definition, we now state such symmetric properties on the smallest non-trivial net CNG1, as mentioned in Theorem 5, as well as the smallest non-trivial net CNG1 with both $M_{\xi}$ and $N_{\xi}$ being odd numbers, as mentioned in Theorem 7 .

Example 4. With regards to the structure of Figure 5, as mentioned in Theorem 5, with $\rho(a)=\rho(b)=$ $\rho(c)=\rho(d)=(1,0,0)$.

Consider the following three automorphisms $f, b, h$ of $\xi_{4,4}$ :

(a) $f(a)=b, f(b)=a, f(c)=d, f(d)=c$,

(b) $\quad b(a)=c, b(b)=d, b(c)=a, b(d)=b$,

(c) $h(a)=d, h(b)=c, h(c)=b, h(d)=a$,

together with $\mathscr{F}$, the trivial automorphism of $\xi$.

As a result, $\xi_{4,4}$ is thus strong point-wise symmetric (SPS) and weak edge-wise symmetric (wES).

Example 5. With regards to the structure of Figure 8, as mentioned in Theorem in Theorem 7 , with $\mathbf{p}+\mathbf{q}+\mathbf{r}=$ $(0,0,0) ;|\mathbf{p}+\mathbf{r}|,|\mathbf{q}+\mathbf{r}| \leq 1$; and $\rho(a)=\rho(b)=\rho(c)=\rho(d)=\rho(e)=(1,0,0)$.

In this case, as non-void vertices having different membership values, only one automorphism of $\xi_{5,7}$, which is the identity mapping $\mathscr{I}: V \rightarrow V$ where $\mathscr{I}(v)=v$ for all $v \in V$. As $\mathscr{I}(a) \neq b, \xi_{5,7}$ is, thus, point-wise asymmetric (PA). It is, nonetheless, weak edge-wise symmetric (wES).

We now give an example of CNG1 which is asymmetric (i.e., both edge-wise and point-wise).

Example 6. $\widetilde{\xi}=\langle V, \rho, \omega\rangle$ has the structure as shown in Figure 25:

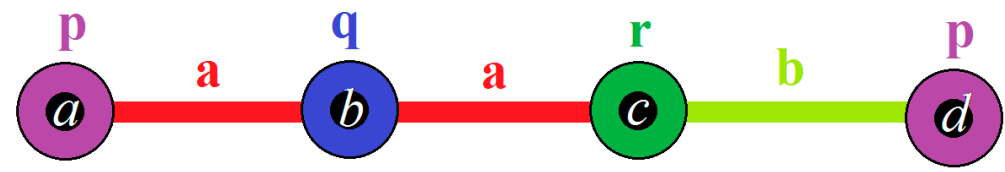

Figure 25. A $\widetilde{\zeta}$ which is both point-wise asymmetric and edge asymmetric, as mentioned in Example 6. 
with $|\{\mathbf{p}, \mathbf{q}, \mathbf{r}\}|=3,|\{\mathbf{a}, \mathbf{b}\}|=2$.

Only the trivial automorphism $\mathscr{I}$ can be formed. As $\mathscr{I}(a) \neq d, \widetilde{\xi}$ is point-wise asymmetric. Moreover, as $\mathscr{I}(a) \neq b, \widetilde{\zeta}$ is edge asymmetric.

We end this section by giving a conjecture, which shall be dealt with in our future work:

Conjecture 1. The smallest non-trivial net CNG1 $\xi$, with both $M_{\xi}$ and $N_{\xi}$ being odd numbers, and is both SPS and wES, must be of the structure as shown in Figure 26:

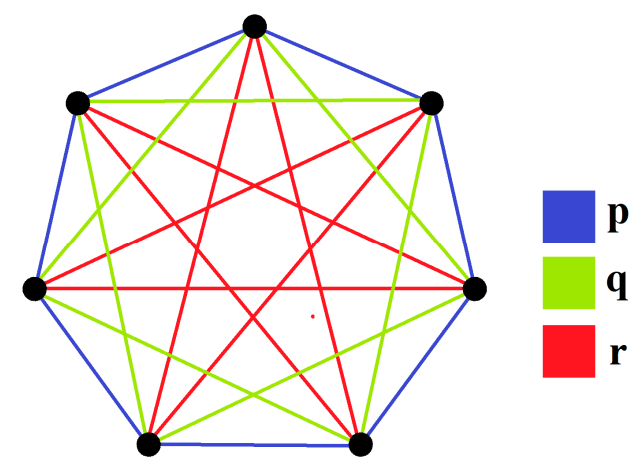

Figure 26. $\xi$ for conjecture 1. $\mathbf{p}+\mathbf{q}+\mathbf{r}=(0,0,0)$.

\section{Conclusions}

In this article, we presented a new concept of the neutrosophic graph called complex neutrosophic graphs of type 1 (CNG1), and also proceeded to present a matrix representation of it.

The strength of CNG1 lies in the presence of both magnitude and direction for the parameters involved, as has been illustrated in Section 3. As the parameters have directions, even when the resultant degree of a vertex is zero, the edges to that vertex need not necessarily be void. Thus the concept of CNG1 may also be used in engineering, such as in metal frameworks, for example in the construction of power lines, so that even when the beams are under tension, the resultant force at a point (possibly being a cornerstone) joining all those beams are zero.

The concept of CNG1 can also be applied to the case of bipolar complex neutrosophic graphs (BCNG1). We have plans to expand on this interesting concept in the near future, and plan to study the concept of completeness, regularity, and CNGs of type 2.

As we can see in Section 6, when the choices of $M_{\xi}$ and $N_{\xi}$ becomes more restrictive, the smallest non-trivial net CNG1 $\xi$ increases in complexity. This makes us wonder what will be the smallest non-trivial net CNG1 $\xi$ in the case when both $M_{\xi}$ and $N_{\xi}$ are not divisible by all primes up to $5(7,11$, etc.), as well as whether their symmetric properties, as outlined in Section 7. However, the proof of such cases will become much more tedious and, therefore, we would have to utilize computer programs, such as MATLAB and SAGE, in order to find those non-trivial net CNG1 $\xi$. Therefore our future research in this area involves plans to deal with those non-trivial net CNG1 $\xi$. We are motivated by the interest to know if there exist some general patterns or general theorems governing such smallest non-trivial net CNG1 as $M_{\xi}$ and $N_{\xi}$ become more restrictive.

We are currently working on developing a more in-depth theoretical framework concerning the symmetric properties of CNG1, and have plans to extend this to other types of fuzzy graphs in the future. We are also motivated by the works presented in [30-32], and look forward to extending our work to other generalizations of neutrosophic sets, such as interval complex neutrosophic sets, and apply the work in medical imaging problems and recommender systems.

Author Contributions: Conceptualization, S.G.Q., S.B., A.B., and M.T.; Methodology, S.B.; Software, S.G.Q.; Validation, G.S., S.B. and S.G.Q.; Formal Analysis, G.S. and F.S.; Writing-Original Draft Preparation, S.B.; Writing-Review \& Editing, G.S. and F.S.; Visualization, S.G.Q.; Supervision, G.S. and F.S. 
Funding: This research was funded by the Ministry of Education, Malaysia under grant no. FRGS/1/2017/STG06/ UCSI/03/1 and UCSI University, Malaysia under grant no. Proj-In-FOBIS-014.

Acknowledgments: The authors would like to thank the Editor-in-Chief and the anonymous reviewers for their valuable comments and suggestions.

Conflicts of Interest: The authors declare no conflict of interest.

\section{References}

1. Smarandache, F. Neutrosophic Probability, Set, and Logic; ProQuest Information \& Learning: Ann Arbor, MI, USA, 1998; 105p.

2. Zadeh, L.A. Fuzzy sets. Inform. Control 1965, 8, 338-353. [CrossRef]

3. Atanassov, K.T. Intuitionistic fuzzy sets. Fuzzy Sets Syst. 1986, 20, 87-96. [CrossRef]

4. Turksen, I. Interval valued fuzzy sets based on normal forms. Fuzzy Sets Syst. 1986, 20, 191-210. [CrossRef]

5. Wang, H.; Smarandache, F.; Zhang, Y.; Sunderraman, R. Single valued neutrosophic sets. Multispace Multistruct. 2010, 4, 410-413.

6. Neutrosophic Set Theory. Available online: http:/ / fs.gallup.unm.edu/NSS/ (accessed on 1 December 2017).

7. Shannon, A.; Atanassov, K. A first step to a theory of the intuitionistic fuzzy graphs. In Proceedings of the First Workshop on Fuzzy Based Expert Systems, Sofia, Bulgaria, 28-30 September 1994; Akov, D., Ed.; 1994; pp. 59-61.

8. Smarandache, F. Symbolic Neutrosophic Theory; Europanova Asbl: Brussels, Belgium, 2015.

9. Smarandache, F. Refined literal indeterminacy and the multiplication law of sub-indeterminacies. Neutrosophic Sets Syst. 2015, 9, 58-63.

10. Smarandache, F. Types of Neutrosophic Graphs and neutrosophic Algebraic Structures together with their Applications in Technology. In Proceedings of the Seminar UniversitateaTransilvania din Brasov, Facultatea de Design de ProdussiMediu, Brasov, Romania, 6 June 2015.

11. Smarandache, F. (Ed.) Neutrosophic overset, neutrosophic underset, neutrosophic offset. In Similarly for Neutrosophic Over-/Under-/Off-Logic, Probability, and Statistics; Pons Editions: Brussels, Belgium, 2016; pp. 158-168.

12. Broumi, S.; Talea, M.; Bakali, A.; Smarandache, F. Single valued neutrosophic graphs. J. New Theory 2016, 10, 86-101.

13. Broumi, S.; Talea, M.; Smarandache, F.; Bakali, A. Single valued neutrosophic graphs: Degree, order and size. In Proceedings of the IEEE International Conference on Fuzzy Systems, Vancouver, BC, Canada, 24-29 July 2016; IEEE: Piscataway, NJ, USA, 2016; pp. 2444-2451.

14. Broumi, S.; Bakali, A.; Talea, M.; Hassan, A.; Smarandache, F. Isolated single valued neutrosophic graphs. Neutrosophic Sets Syst. 2016, 11, 74-78.

15. Smarandache, F. (Ed.) Nidus idearum. In Scilogs, III: Viva la Neutrosophia; Pons asbl: Brussels, Belgium, 2017; ISBN 978-1-59973-508-5.

16. Samanta, S.; Sarkar, B.; Shin, D.; Pal, M. Completeness and regularity of generalized fuzzy graphs. SpringerPlus 2016, 5, 1-14. [CrossRef] [PubMed]

17. Broumi, S.; Bakali, A.; Talea, M.; Hassan, A.; Smarandache, F. Generalized single valued neutrosophic graphs of first type. In Proceedings of the 2017 IEEE International Conference on Innovations in Intelligent Systems and Applications (INISTA), Gdynia Maritime University, Gdynia, Poland, 3-5 July 2017; pp. 413-420.

18. Ramot, D.; Menahem, F.; Gideon, L.; Abraham, K. Complex Fuzzy Set. IEEE Trans. Fuzzy Syst. 2002, 10, 171-186. [CrossRef]

19. Selvachandran, G.; Maji, P.K.; Abed, I.E.; Salleh, A.R. Complex vague soft sets and its distance measures. J. Intell. Fuzzy Syst. 2016, 31, 55-68. [CrossRef]

20. Selvachandran, G.; Maji, P.K.; Abed, I.E.; Salleh, A.R. Relations between complex vague soft sets. Appl. Soft Comp. 2016, 47, 438-448. [CrossRef]

21. Kumar, T.; Bajaj, R.K. On complex intuitionistic fuzzy soft sets with distance measures and entropies. J. Math. 2014, 2014, 1-12. [CrossRef]

22. Alkouri, A.; Salleh, A. Complex intuitionistic fuzzy sets. AIP Conf. Proc. 2012, 1482, 464-470.

23. Selvachandran, G.; Hafeed, N.A.; Salleh, A.R. Complex fuzzy soft expert sets. AIP Conf. Proc. 2017, 1830, 1-8. [CrossRef] 
24. Selvachandran, G.; Salleh, A.R. Interval-valued complex fuzzy soft sets. AIP Conf. Proc. 2017, 1830, 1-8. [CrossRef]

25. Greenfield, S.; Chiclana, F.; Dick, S. Interval-valued Complex Fuzzy Logic. In Proceedings of the IEEE International Conference on Fuzzy Systems, Vancouver, BC, Canada, 24-29 July 2016; IEEE: Piscataway, NJ, USA, 2016; pp. 1-6.

26. Ali, M.; Smarandache, F. Complex neutrosophic set. Neural Comp. Appl. 2017, 28, 1817-1834. [CrossRef]

27. Thirunavukarasu, P.; Suresh, R.; Viswanathan, K.K. Energy of a complex fuzzy graph. Int. J. Math. Sci. Eng. Appl. 2016, 10, 243-248.

28. Smarandache, F. A geometric interpretation of the neutrosophic set-A generalization of the intuitionistic fuzzy set. In Proceedings of the IEEE International Conference on Granular Computing, National University of Kaohsiung, Kaohsiung, Taiwan, 8-10 November 2011; Hong, T.P., Kudo, Y., Kudo, M., Lin, T.Y., Chien, B.C., Wang, S.L., Inuiguchi, M., Liu, G.L., Eds.; IEEE Computer Society; IEEE: Kaohsiung, Taiwan, 2011; pp. 602-606.

29. Smarandache, F. Degree of dependence and independence of the (sub) components of fuzzy set and neutrosophic set. Neutrosophic Sets Syst. 2016, 11, 95-97.

30. Ali, M.; Son, L.H.; Khan, M.; Tung, N.T. Segmentation of dental X-ray images in medical imaging using neutrosophic orthogonal matrices. Expert Syst. Appl. 2018, 91, 434-441. [CrossRef]

31. Ali, M.; Dat, L.Q.; Son, L.H.; Smarandache, F. Interval complex neutrosophic set: Formulation and applications in decision-making. Int. J. Fuzzy Syst. 2018, 20, 986-999. [CrossRef]

32. Ali, M.; Son, L.H.; Thanh, N.D.; Minh, N.V. A neutrosophic recommender system for medical diagnosis based on algebraic neutrosophic measures. Appl. Soft Comp. 2017. [CrossRef]

(C) 2018 by the authors. Licensee MDPI, Basel, Switzerland. This article is an open access article distributed under the terms and conditions of the Creative Commons Attribution (CC BY) license (http://creativecommons.org/licenses/by/4.0/). 